\title{
Therapeutic Targeting of Redox Signaling in Myofibroblast Differentiation and Age-Related Fibrotic Disease
}

\author{
Natalie Sampson, ${ }^{1}$ Peter Berger, ${ }^{2}$ and Christoph Zenzmaier ${ }^{2}$ \\ ${ }^{1}$ Department of Experimental Urology, Medical University of Innsbruck, 6020 Innsbruck, Austria \\ ${ }^{2}$ Institute for Biomedical Aging Research, Austrian Academy of Sciences, 6020 Innsbruck, Austria
}

Correspondence should be addressed to Peter Berger, peter.berger@oeaw.ac.at

and Christoph Zenzmaier, christoph.zenzmaier@oeaw.ac.at

Received 29 June 2012; Accepted 18 September 2012

Academic Editor: Paula Ludovico

Copyright (c) 2012 Natalie Sampson et al. This is an open access article distributed under the Creative Commons Attribution License, which permits unrestricted use, distribution, and reproduction in any medium, provided the original work is properly cited.

Myofibroblast activation plays a central role during normal wound healing. Whereas insufficient myofibroblast activation impairs wound healing, excessive myofibroblast activation promotes fibrosis in diverse tissues (including benign prostatic hyperplasia, $\mathrm{BPH}$ ) leading to organ dysfunction and also promotes a stromal response that supports tumor progression. The incidence of impaired wound healing, tissue fibrosis, $\mathrm{BPH}$, and certain cancers strongly increases with age. This paper summarizes findings from in vitro fibroblast-to-myofibroblast differentiation systems that serve as cellular models to study fibrogenesis of diverse tissues. Supported by substantial in vivo data, a large body of evidence indicates that myofibroblast differentiation induced by the profibrotic cytokine transforming growth factor beta is driven by a prooxidant shift in redox homeostasis due to elevated production of NADPH oxidase 4 (NOX4)-derived hydrogen peroxide and supported by concomitant decreases in nitric oxide/cGMP signaling and reactive oxygen species (ROS) scavenging enzymes. Fibroblast-to-myofibroblast differentiation can be inhibited and reversed by restoring redox homeostasis using antioxidants or NOX4 inactivation as well as enhancing nitric oxide/cGMP signaling via activation of soluble guanylyl cyclases or inhibition of phosphodiesterases. Current evidence indicates the therapeutic potential of targeting the prooxidant shift in redox homeostasis for the treatment of age-related diseases associated with myofibroblast dysregulation.

\section{Myofibroblast Biology}

The myofibroblast is a specialized cell type that combines the extracellular matrix (ECM)-producing characteristics of fibroblasts with the cytoskeletal and contractile properties of smooth muscle cells (SMCs) as reviewed recently [1]. Defined by (i) their de novo expression of alpha-smooth muscle cell actin ( $\alpha$-SMA, encoded by the gene ACTA2) in stress fibers and (ii) contractile force, myofibroblasts play a critical role during normal wound healing and thereby in maintaining tissue integrity [2]. In addition, myofibroblasts secrete growth factors that attract epithelial cells for subsequent wound closure (reepithelialization) [3-5]. Normal tissue function and architecture is restored upon completion of reepithelialization via massive apoptosis of myofibroblasts and vascular cells followed by their subsequent clearance from the wound site [6]. Whilst relatively poorly understood, induction of cellular senescence via telomere-dependent and -independent mechanisms may also facilitate completion of wound healing [7-10]. For example, upon tissue injury telomerase activation is thought to enable cell proliferation for repair of local tissue damage [9]. However, its subsequent downregulation appears to be required for downstream induction of cellular senescence, cessation of wound healing, and cell clearance by the immune system $[7,10]$.

Dysregulation of the wound healing response has significant pathological consequences. On the one hand, a major clinical problem in the elderly is impaired wound healing, whereby wound repair is temporally delayed and all phases of wound healing exhibit characteristic changes 
[11]. By contrast, excessive and/or persistent myofibroblast activity results in continued synthesis of ECM, dysregulation of growth factor signaling and consequently to tissue fibrosis and organ dysfunction [12]. This paper focuses on growing evidence indicating that redox signaling downstream of dysregulated transforming growth factor beta (TGF $\beta$ ) promotes myofibroblast differentiation and the development/progression of several age-related fibrotic diseases. Findings from cellular fibrosis models are highlighted, which have improved our understanding of the molecular mechanisms underlying such disorders.

\section{Myofibroblast Origin and Induction}

Although cell types other than fibroblasts, (e.g., vascular SMCs, pericytes, bone marrow-derived fibrocytes, resident epithelial cells via epithelial-to-mesenchymal transition, and endothelial cells via endothelial-to-mesenchymal transition) have been reported to undergo differentiation into myofibroblasts in vitro, the extent of their contribution to the in vivo myofibroblast pool remains controversial [12, 13]. Rather, it is widely considered that myofibroblasts predominantly originate from the differentiation of local tissue fibroblasts [13].

Following injury or during chronic inflammation, fibroblast-to-myofibroblast differentiation occurs via a two-step process, initiated by changes in mechanical tension of the ECM that are transmitted to the fibroblast cytoskeleton via RhoA/ROCK signaling [14]. Consequently, fibroblasts adopt an "activated" phenotype (referred to as "protomyofibroblast") and deposit new ECM components [15]. Soluble factors and cytokines produced by platelets and infiltrating leukocytes play a major role in the differentiation to the $\alpha$-SMA-expressing myofibroblast phenotype. In particular, the combined action of the splice variant ED-A of cellular fibronectin and TGF $\beta$, especially TGF $\beta 1$, that initially is secreted by platelets and phagocytic cells at the wound site [15]. However, proto-myofibroblasts and myofibroblasts also secrete and activate TGF $\beta 1$ thereby generating an autocrine feed-forward loop further driving myofibroblast differentiation $[3,16]$.

TGF $\beta 1$ is a key inducer of myofibroblast differentiation in cells of diverse histological origin, including breast, skin, prostate, kidney, heart, lung, and liver [17-23]. TGF $\beta 1$ exerts its effects via transcriptional events downstream of Smad2/3 activation and Smad-independent regulation of mitogenactivated protein kinase (MAPK) and PI3 kinase/Akt pathways $[1,3,24]$. Collectively, activation of these pathways results in the deposition of ECM and secretion of paracrineand autocrine-acting growth factors $[3,25]$. Importantly, the ECM can directly bind to and release growth factors, such as heparan sulfate binding to fibroblast growth factor 2 [26]. Such interactions can serve to sequester and protect growth factors from degradation and/or enhance their activity [27]. In addition, indirect interactions are required for signal transduction of some growth factors, for example, integrin binding is necessary for induction of angiogenesis by vascular endothelial cell growth factor [28]. Thus, maintaining ECM homeostasis is critical to regulate not only tissue architecture but also cellular signaling cascades.

\section{Aging, Dysregulation of Myofibroblast Differentiation, and Fibrosis}

Dysregulation of the wound healing response, particularly in association with chronic inflammation and injury (e.g., in the liver due to viral infection, in the lung from chronic obstructive pulmonary disease, in the heart following myocardial infarction) can result in excessive myofibroblast activation and organ fibrosis [29]. It is perhaps therefore not surprising that the incidence of many fibrosis-associated diseases increases sharply with advancing age, for example, cardiovascular disease, fibrosis of the liver, lung and kidney, and benign prostatic hyperplasia (BPH), a classic agerelated fibrotic-like disease characterized by fibroblast to myofibroblast differentiation and ECM deposition [24, 3035]. Fibrosis is also observed in the stromal response to many tumors, including liver and prostate cancer, both of whose incidence is strongly linked with aging and chronic inflammation [36-39]. Myofibroblasts in the tumor-adjacent stroma (termed "reactive stroma") not only actively promote tumorigenesis and angiogenesis via ECM deposition and mitogen secretion but also support cancer cell invasion and metastasis by producing ECM remodeling enzymes [40-44].

Tissue and organ fibrosis are thought to arise from failure of the wave of myofibroblast apoptosis during wound healing, resulting in persistent myofibroblast activation, excessive ECM deposition, altered growth factor signaling and cellular proliferation $[12,45]$. In addition, failure of the timely induction of cellular senescence and dysregulation of telomere biology may also lead to organ fibrosis [7-10]. Consistently, shortened telomeres and telomerase mutations have been observed in familial and idiopathic pulmonary fibrosis $[46,47]$. However, cell senescence also ameliorates fibrosis via telomere-independent mechanisms [8] and may partially explain discrepancies observed in rodent fibrosis models, whereby telomere deficiency had no effect on chemical-induced fibrosis [48]. The contribution of cellular senescence and telomere biology to age-related fibrosis pathologies remains to be fully investigated.

Given its potent myofibroblast differentiating effects and ability to promote myofibroblast survival in an autocrine manner, TGF $\beta 1$ is considered a key molecule underlying the pathophysiology of fibrotic disease $[29,49,50]$. Interestingly, the TGF $\beta$ signaling intermediate Smad 3 is a direct repressor of telomerase reverse transcriptase $[51,52]$, a key enzyme required for telomerase activity, suggesting that autocrine TGF $\beta$ signaling by myofibroblasts may override the protective mechanism of cellular senescence further exacerbating tissue fibrosis. Consistent with its central role in ageassociated fibrogenic pathologies, elevated TGF $\beta 1$ levels and signaling are observed in $\mathrm{BPH}$ and preneoplastic prostatic lesions, tumor-associated reactive stroma, cardiovascular remodeling, renal interstitial fibrosis, chronic obstructive pulmonary disease, idiopathic pulmonary fibrosis (IPF), and in chronic liver disease [44-52]. 


\section{Cellular versus Animal Model Systems for Studying Age-Associated Fibrosis}

Several animal models for fibrotic diseases such as systemic sclerosis, pulmonary, liver, cardiac, and renal fibrosis have been established [53-58]. Whilst such models can be valuable tools to understand disease pathology and evaluate novel therapeutic strategies, fibrosis does not normally develop spontaneously in these animals and typically has to be artificially induced, for example by chemical means [53-58]. Moreover, there may be species-specific differences in organ anatomy, physiology, and disease susceptibility. A notable example is the prostate gland $[32,59,60]$. Significant anatomical differences between rodents and humans also have to be considered in other organs for example, lung and skin $[61,62]$. In addition, whilst favorable for experimental reproducibility, the use of genetically identical inbred strains does not recapitulate the heterogeneity of human pathologies. Furthermore, animal models are costly and time-consuming and thus not suitable for high throughput drug screening.

Fibrotic diseases are often associated with inflammation, thus it is difficult to dissect if fibrosis suppression in animal models is due to direct antifibrotic effects of a drug or due to indirect anti-inflammatory effects. Cellular in vitro model systems to investigate specific cellular or molecular responses can be very useful to elucidate inflammation-independent antifibrotic targets. Moreover, cellular in vitro models offer the significant advantage that human-derived cells can be analyzed. In addition, they are less costly, deliver more rapid results than animal models, can be easily genetically manipulated and are amenable for high throughput screening.

Numerous in vitro models of fibrosis have been successfully devised. In vitro culture of fibroblasts in 3D collagen-gel matrices leads to progressive contraction of the gel over the course of several days mimicking wound contraction [63]. This model has been widely used to investigate the contractility of fibroblasts/myofibroblasts derived from various tissues affected by fibrotic disease including skin, mucosa, lung, cornea, and heart [64-71].

Overwhelming in vitro and in vivo data demonstrate the central role of TGF $\beta 1$ and myofibroblast differentiation in the etiology of diverse fibrotic disorders (see above). Thus, an in vitro approach successfully employed by us and others to model fibroblast-to-myofibroblast differentiation in $\mathrm{BPH}$ and prostate cancer reactive stroma applies TGF $\beta 1$ to primary prostatic fibroblasts, which induces their robust differentiation into myofibroblasts $[3,25,39,72,73]$ (Figure 1). This approach has also been extensively employed to model myofibroblast differentiation of hepatic stellate cells and of fibroblasts from breast, skin, kidney, heart, and lung [20, 21, 23, 74-77]. Myofibroblast differentiation can be subsequently monitored at the molecular level by the induction of molecular markers such as $\alpha$-SMA and collagens as well as at the morphological level whereby the thin and elongated phenotype characteristic of fibroblasts changes to the flattened, less light refractive myofibroblast phenotype, which is accompanied by the appearance of contractile actin bundles (Figure 1). The validity of this in vitro model system is highlighted by numerous molecular and cellular parallels with animal models and patient specimens that collectively reveal a central role of dysregulated redox signaling by NADPH oxidase 4 (NOX4) and nitric oxide (NO)/cyclic guanosine monophosphate (cGMP) in the development of age-related fibrotic disease.

\section{NADPH Oxidase-Derived Reactive Oxygen Species in the Regulation of Myofibroblast Differentiation}

Accumulation of nonspecific oxidative damage by high levels of free radicals is thought to be a major contributor to organismal aging [78]. However, when produced in a regulated manner, reactive oxygen species (ROS), NO, and reactive nitrogen species (RNS) can act as biological second messengers in a variety of signal transduction pathways, including myofibroblast differentiation [79].

The NADPH oxidase (NOX) enzyme family, comprising seven members, catalyzes the transfer of electrons across biological membranes from NADPH to oxygen thereby generating superoxide $\left(\mathrm{O}_{2}{ }^{--}\right)$[80]. The NOX family is thereby unique as ROS production is their primary function and not a byproduct as is the case for other ROS-producing enzyme systems, such as xanthine oxidase, mitochondrial respiratory chain, lipid peroxidases, or uncoupled endothelial NO synthase [81].

NOX4 is unique among NOX enzymes in that it is constitutively active with primary regulation occurring at the transcriptional level $[82,83]$. Moreover, whilst other NOX enzymes produce superoxide, NOX4 is associated with constitutive hydrogen peroxide $\left(\mathrm{H}_{2} \mathrm{O}_{2}\right)$ production, however whether this occurs via superoxide dismutation or direct $\mathrm{H}_{2} \mathrm{O}_{2}$ production remains controversial [82, 84, 85]. The greater stability but lower reactivity of $\mathrm{H}_{2} \mathrm{O}_{2}$ compared to superoxide is consistent with a signaling function of NOX4derived ROS as underscored by observations that sustained and elevated NOX4-derived ROS levels induced by cytokines and growth factors do not induce oxidative damage [3, 86, 87].

Critically, several growth factors, in particular TGF $\beta 1$, and stimuli implicated in the pathogenesis of fibrotic disease induce NOX4 expression in diverse cell types as reviewed recently [88]. Consequently, NOX4-derived ROS have been implicated in the pathophysiology of fibrotic disorders, including $\mathrm{BPH}$, IPF, cardiac remodeling, renal, and liver fibrosis, tumorigenesis and the stromal response to prostate, breast and liver cancers [3, 20, 74, 89, 90].

Using in vitro model systems, NOX4-derived ROS have been shown to be essential downstream inducers of TGF $\beta 1$ mediated myofibroblast differentiation in a myriad of cell types of diverse histological origin. For example, we demonstrated using NOX4 silencing and antioxidants that induction of NOX4-derived ROS in response to TGF $\beta 1$ drives myofibroblast differentiation of prostatic fibroblasts [3]. Similarly, TGF $\beta 1$-induction of NOX4-derived ROS was required for fibroblast-to-myofibroblast differentiation in in vitro models of cardiac, pulmonary, renal, and adventitial fibrosis [20, 21, 74, 75, 91]. Besides inducing differentiation 

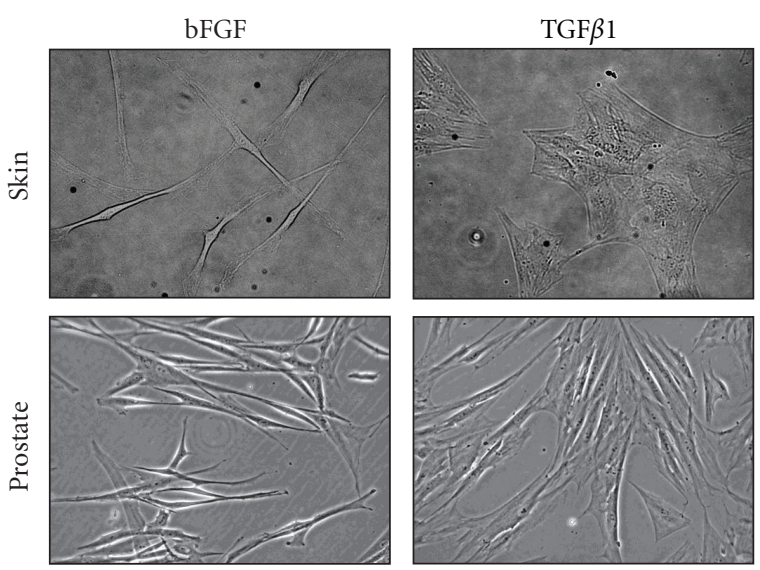

(a)
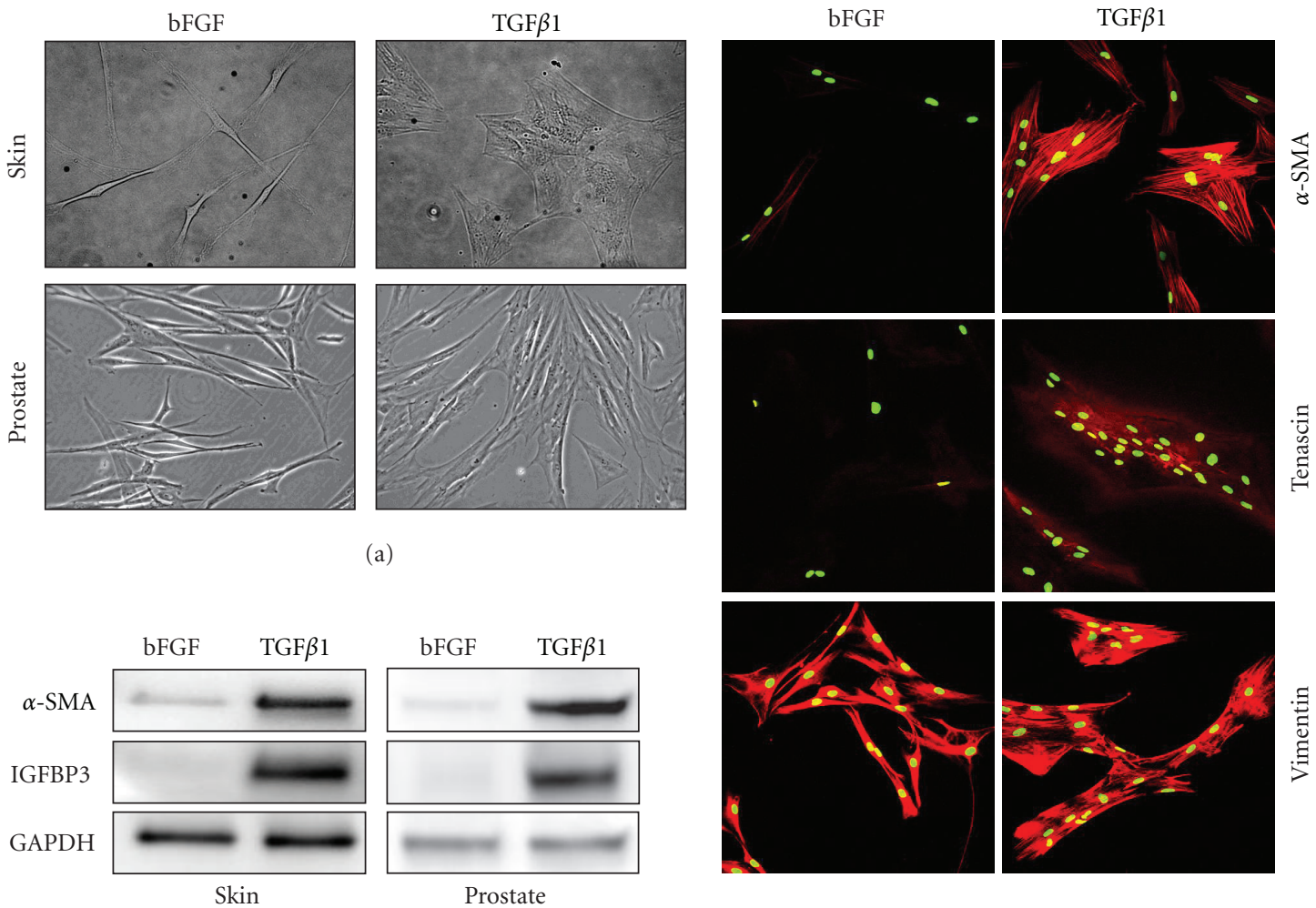

(b)

(c)

Figure 1: In vitro modeling of fibroblast-to-myofibroblast differentiation. Fibroblast-to-myofibroblast differentiation is induced following incubation of fibroblasts for at least 24 hours with $1 \mathrm{ng} / \mathrm{mL}$ transforming growth factor beta 1 (TGF $\beta 1$ ) in RPMI-1640 media supplemented with $1 \%$ steroid hormone-depleted charcoal-treated bovine calf serum (ctBCS). Steroid hormone depletion is particularly important for fibroblasts derived from endocrine tissues such as the prostate, since androgens attenuate TGF $\beta$ signaling and vice versa [32]. Similar to commercial formulations of fibroblast growth media, the fibroblast phenotype of mock control cells is maintained with $1 \mathrm{ng} / \mathrm{mL}$ basic fibroblast growth factor (bFGF) and serves to inhibit differentiation-inducing stimuli (e.g., TGF $\beta$ ) in serum. Myofibroblast differentiation can be monitored at the morphological level by phase contrast microscopy (a) and molecular level by Western blotting (b) or immunofluorescence (c). (a) Primary human dermal fibroblasts (top left) and prostatic fibroblasts (lower left) exhibit a typical thin, elongated, and light refractive phenotype, whereas upon differentiation with TGF $\beta 1$ for 24 hours dermal (top right) and prostatic myofibroblasts (lower right) display a flattened, less light refractive phenotype. (b) TGF $\beta 1$ induces the expression of myofibroblasts markers $\alpha$-smooth muscle cell actin ( $\alpha$-SMA) and insulin-like growth factor binding protein 3 (IGFBP3) [25] in primary human dermal (left) and prostatic (right) fibroblasts as determined by Western blotting. GAPDH served as loading control. (c) TGF $\beta 1$ treatment of primary human prostatic fibroblasts induces the expression of myofibroblast markers tenascin and filamentous $\alpha$-SMA as determined by immunofluorescence and confocal laser scanning microscopy. Both myofibroblasts and fibroblasts express the mesenchymal marker vimentin. Nuclei were counterstained with SytoxGreen. (c) Adapted from [25], used with permission.

of fibroblasts, NOX4 was shown to play a role in TGF $\beta 1$ mediated cytoskeletal remodeling of vascular endothelial cells and in maintaining the differentiated phenotype of vascular SMCs [92, 93]. Moreover, induction of NOX4 by TGF $\beta 1$ is required for hepatic stellate cell activation and their subsequent transdifferentiation into myofibroblasts [22]. Interestingly, NOX4 induction was also required for insulin-induced adipocyte differentiation of preadipocytes [94]. Collectively, these data suggest a broader role of NOX4 in regulating differentiation in response to changes in the cellular environment.

These findings from in vitro fibroblast-to-myofibroblast differentiation model systems are supported by several in vivo data. We demonstrated that NOX4 mRNA levels specifically correlated with the myofibroblast phenotype in benign prostatic tissue [3]. NOX4 expression was higher in pulmonary fibroblasts from patients with IPF compared with controls and correlated with mRNA levels of the myofibroblast markers $\alpha$-SMA and procollagen I $\alpha 1$ [75]. Consistently, NOX4 is expressed in situ in fibroblastic foci in the lung of IPF patients and two mouse models of pulmonary fibrosis. Moreover, targeting NOX4 via siRNA or a nonspecific NOX inhibitor diphenylene iodonium attenuated lung fibrosis in two murine models of lung injury [74]. NOX4 may also be involved in vascular remodeling associated with IPF [95]. In animal models of diabetic nephropathy, treatment of diabetic rats with NOX4 siRNAs attenuated renal fibrosis strongly implicating a causative role of NOX4-derived ROS in the fibrogenic response to renal injury [96]. Recently, high levels of NOX4 were found in liver biopsy samples from patients with autoimmune hepatitis, which colocalized with $\alpha$-SMA. Moreover, liver fibrosis could be attenuated in 
mouse models via genetic deletion of NOX4 or application of a dual NOX1/NOX4 inhibitor GKT137831 [22], indicating a direct role of NOX4 in the pathogenesis of fibrosis.

NOX4 induction does not appear to contribute to fibrogenesis via direct oxidative stress but rather by chronic dysregulation of downstream signaling pathways. NOXderived ROS mediate their signaling functions via reversible oxidation of thiol groups of low $\mathrm{pKa}$ cysteine residues in target proteins, including transcription factors, MAPKs, protein tyrosine phosphatases (PTPs), and protein tyrosine kinases (PTKs) [87]. Typically, thiol oxidation results in PTP inactivation but kinase activation thus promoting phosphorylation signaling cascades [87]. Only a small proportion of cysteines exhibit the necessary close proximity to basic amino acids to undergo transition to a sulfenic acid, thereby providing a basis for specificity of thiol redox signaling which reversibly regulates biological function by (i) chemically altering active site cysteines, (ii) altering macromolecular interactions, and (iii) modifying allosteric Cys [97].

The precise oxidative target(s) of NOX4-derived ROS that culminate in myofibroblast differentiation in response to TGF $\beta$ remain largely unknown. However, in lung fibroblasts TGF $\beta 1$ induced NOX4-derived ROS directly oxidatively inactivate MKP1, a nuclear dual-specificity MAPK phosphatase that targets JNK, and p38, leading to sustained activation of JNK and p38 MAPKs [98]. Similarly, in prostatic fibroblasts, NOX4-derived ROS were required for sustained phosphorylation of JNK whose activity in turn was essential for downstream $\alpha$-SMA induction, and myofibroblast differentiation [3]. By contrast, NOX4 mediates TGF $\beta$-induced myofibroblast differentiation of renal fibroblasts via ERK1/2 [21], whereas angiotensin II-induction of NOX4-derived ROS and fibronectin/ECM deposition in renal mesangial cells occurred via Src activation [99]. Thus, it is likely that the oxidative target(s) of NOX4-derived ROS are tissue-, celltype, and/or context-specific.

In summary, whilst acute induction of NOX4 may be beneficial in inducing the myofibroblast phenotype for wound repair, the persistence of myofibroblasts together with autocrine TGF $\beta$ signaling may result in chronic NOX4 activation and dysregulation of signaling pathways culminating in myofibroblast differentiation, fibrosis, and organ dysfunction.

The cellular redox status and thus signaling potential of NOX4-derived ROS is regulated by antioxidant systems. Concomitant to TGF $\beta 1$-mediated induction of NOX4 during prostatic myofibroblast differentiation, a number of ROSscavenging enzymes were downregulated, including the selenium transporter SEPP1 and selenium (Se)-containing ROS scavenging enzymes such as glutathione peroxidase 3 (GPX3) and thioredoxin reductase 1 (TXNRD1) [3]. The essential trace element Se is an integral component of GPX3 and TXNRD1 enzymes being incorporated as selenocysteine (Sec) at their active site and is critical for correct protein folding/function [100]. Consistent with the role of SEPP1 in delivering Se to peripheral tissues for selenoprotein biosynthesis [101, 102], supplementation of prostatic fibroblasts with exogenous Se restored expression of GPX3 and TXNRD1 as well as TXNRD1 enzyme activity, depleted
TGF $\beta 1$-induced ROS downstream of NOX4 induction and inhibited myofibroblast differentiation [3].

Given the central role of elevated TGF $\beta 1$ in fibrogenesis and that SEPP1 is a direct transcriptionally-suppressed target of TGF $\beta 1[103,104]$, it is plausible that dysregulation of Sedependent antioxidant systems occurs not only in BPH and the stromal response to prostate cancer but also in other fibrotic disorders. Indeed, the antifibrotic potential of Se is not restricted to prostatic stromal cells since exogenous Se also inhibited TGF $\beta$-mediated myofibroblast transdifferentiation of hepatic stellate cells [105]. Moreover, in a rat model of thyroid fibrosis, Se deficiency promoted thyroid fibrosis in a TGF $\beta$-dependent manner [106], whereas Se supplementation decreased hepatic fibrosis in mice [107]. Consistent with in vitro findings, SEPP1 was specifically lost in the periglandular tumor-associated stroma of prostate cancer patients [3]. These findings are consistent with a large body of animal and human clinical data that Se deficiency or supplementation increases or reduces tumor incidence, respectively [108-111]. Moreover, a recent dose-response meta-analysis revealed that overall prostate cancer risk was 15-25\% (for advanced PCa: 40-50\%) lower in men with plasma/serum Se levels between 135-170 ng/mL compared with $60 \mathrm{ng} / \mathrm{mL}$ [112].

Collectively, data from in vitro fibroblast-to-myofibroblast differentiation models together with in vivo findings indicate that myofibroblast differentiation in fibrotic disorders and tumor-reactive stroma is driven by a prooxidant shift in intracellular redox signaling caused by elevated ROS and/or reduced antioxidative potential. NOX4 appears to be the major source of elevated ROS and central mediator of TGF $\beta$-induced myofibroblast differentiation in diverse tissues. Thus, restoring cellular redox homeostasis, for example by (i) targeting NOX4, (ii) Se supplementation, and/or (iii) application of antioxidants may represent a promising therapeutic strategy for fibrotic disease.

\section{Nitric Oxide/cGMP Signaling in the Regulation of Myofibroblast Differentiation}

The free radical NO is an important signaling molecule in a variety of biological processes. In vivo NO is biosynthesized from L-arginine by nitric oxide synthases (NOS), involving the oxidation of NADPH and the reduction of molecular oxygen. NO activates soluble guanylyl cyclase (sGC), which generates the second messenger cGMP. cGMP exerts multiple effects, for example it regulates cGMP-dependent protein kinases such as protein kinase G (PKG), cyclic nucleotide phosphodiesterases (PDEs), and cation channels and may have other unknown effects [113].

PDEs comprise a superfamily of phosphohydrolases that regulate cellular CGMP and cyclic adenosine monophosphate (cAMP) levels. PDE type 5 (PDE5) specifically hydrolyzes cGMP and is the major therapeutic target in erectile dysfunction (ED), whereby PDE5 inhibitors increase intracellular cGMP levels to enhance NO/cGMP signaling and thereby promote vasodilation [114].

Besides treatment of ED, PDE5 inhibitors are employed in the treatment of pulmonary arterial hypertension and 
BPH [115, 116]. Noticeably, patients treated with PDE5 inhibitors for ED exhibited beneficial effects on lower urinary tract symptoms (LUTS) secondary to BPH $[117,118]$. These effects were thought to be due to changes in prostatic smooth muscle tone [119-121]. However, our data implicate a direct role of PDE5, which is predominantly expressed in the stromal compartment of the prostate in vivo, and NO/cGMP signaling in myofibroblast differentiation [73]. Pharmacological or genetic inhibition of PDE5 significantly attenuated TGF $\beta 1$-induced myofibroblast differentiation of prostatic fibroblasts in vitro, indicating that enhancing intracellular cGMP levels inhibits myofibroblast differentiation [73]. Consistently, stimulating the generation of intracellular cGMP by the soluble NO donor sodium nitroprusside (SNP) dose-dependently inhibited TGF $\beta 1$-induced differentiation and additional blocking of cGMP hydrolysis by the PDE5 inhibitor tadalafil synergistically enhanced this effect [73].

These findings are consistent with numerous studies implicating an inhibitory role of the NO/cGMP pathway in fibroblast-to-myofibroblast differentiation in other tissues. On the one hand, treatment of human dermal fibroblasts with TGF $\beta 1$ significantly reduced NOS activity and NO levels, whereas restoring cGMP signaling downstream of NOS using SNP and the cell-permeable cGMP analog 8bromo-cGMP significantly suppressed TGF $\beta 1$-induced collagen production [122]. Furthermore, the NOS inhibitor $N_{\omega}$-nitro-L-arginine methyl ester (L-NAME) synergistically potentiated TGF $\beta 1$-induced collagen production in dermal fibroblasts [122]. In addition, increasing cGMP levels using the PDE5 inhibitor sildenafil alone or in combination with the sGC activator BAY 58-2667 attenuated myofibroblast differentiation of fibroblasts from human Peyronie's disease plaques or lung, respectively $[23,123]$. In a similar approach, 8-bromo-cGMP inhibited TGF $\beta 1$-induced myofibroblast differentiation of cardiac fibroblasts isolated from wild-type mice [76]. Likewise, the sGC stimulator BAY 41-2272 elevated intracellular cGMP levels and inhibited myofibroblast differentiation in cardiac fibroblasts [77] and dermal fibroblasts from healthy individuals and patients with systemic sclerosis [124].

In addition to the documented improvement of LUTS secondary to $\mathrm{BPH}$ by PDE5 inhibitors $[117,118]$, beneficial effects of enhancing NO/cGMP signaling have also been reported in several fibrosis models in vivo. For example, attenuating NO signaling by inhibition of inducible NOS (iNOS) activity in rats with TGF $\beta 1$-induced fibrotic plagues of tunica albuginea (a model of Peyronie's disease) resulted in increased myofibroblast abundance and collagen I synthesis in the plaques [125]. Similarly, fibrosis in the penile corpora cavernosa upon streptozotocin-induced diabetes was intensified in iNOS knockout mice compared with wild type [126]. Consistently, stimulating NOS activity in rats via oral administration of the NOS substrate L-arginine resulted in an $80-95 \%$ reduction in both plaque size and collagen: fibroblast ratio in PD-like plaques induced by TGF $\beta 1$ [123]. Similar effects were observed in parallel groups that received the competitive nonselective PDE inhibitor pentoxifylline or PDE5-selective inhibitor sildenafil [123]. Likewise, promoting $\mathrm{NO}$ synthesis via administration of the
NOS substrate L-arginine, significantly elevated endothelial NOS expression but decreased TGF- $\beta 1$ expression and ultimately ameliorated renal interstitial fibrosis, which was markedly aggravated by L-NAME administration in rats with unilateral ureteral obstruction [127].

Alternative approaches to increase cGMP production and NO/cGMP signaling by stimulating sGC activity exhibit similar antifibrotic effects. The sGC stimulator BAY 412272 significantly limited progression of anti-Thy-1-induced chronic renal fibrosis in rats [128]. Likewise, BAY 41-2272 reduced the number of myofibroblasts and decreased collagen accumulation in hypertension-induced cardiac fibrosis in rats [77]. Another sGC stimulator riociguat (BAY 632521) exerted similar inhibitory effects on cardiac and renal interstitial fibrosis in two rat models of hypertension [129] and attenuated fibrotic tissue remodeling in the myocardium and renal cortex of Dahl salt-sensitive rats [130]. Furthermore, BAY 41-2272 prevented the development of bleomycin-induced dermal fibrosis and skin fibrosis in Tsk-1 mice [124]. Interestingly, NO-independent activation of sGC by BAY 60-2770 also attenuated liver fibrosis in rats [131].

Many studies investigating NO/cGMP signaling in fibrotic disease exploit the specific hydrolytic activity of PDE5 for cGMP to indirectly enhance cGMP levels and thereby promote NO signaling. However, an active role of PDE5 in fibrogenesis via altered enzyme activity has also been reported. Glomerular PDE5 expression was increased during antiThyl-induced mesangial proliferative glomerulonephritis in the rat kidney in vivo, and PDE5 inhibition by vardenafil increased glomerular cGMP levels leading to subsequent inhibition of mesangial cell proliferation and ECM accumulation [132].

Collectively, findings from in vitro and in vivo model systems indicate that myofibroblast differentiation is associated with reduced NO/cGMP signaling, suggesting the potential therapeutic benefit of enhancing NO/cGMP signaling (by stimulating sGC activity, and/or preventing cGMP degradation via PDE5 inhibition) in fibrotic disease.

\section{Potential Interplay of ROS and NO/cGMP Signaling in Regulating Myofibroblast Differentiation}

NOX4-derived ROS play a key role in driving myofibroblast differentiation in response to TGF $\beta 1$, whereas increasing NO/cGMP signaling attenuates TGF $\beta 1$-induced fibroblastto-myofibroblast differentiation. This raises an interesting possibility that crosstalk between NO/cGMP and NOX4derived ROS signaling may coordinately regulate myofibroblast differentiation.

Crosstalk between superoxide and NO signaling has been extensively documented, in particular the ability of superoxide to reduce NO levels by direct chemical scavenging or by NOS uncoupling. For example, superoxide can react with $\mathrm{NO}$ generating peroxynitrite $\left(\mathrm{ONOO}^{-}\right)$, thereby depleting NO levels [133]. In addition, superoxide can oxidize the critical nitric oxide synthase (NOS) cofactor tetrahydrobiopterin $\left(\mathrm{BH}_{4}\right)$ leading to NOS uncoupling, which 
results in superoxide generation rather than $\mathrm{NO}$ production [134]. However, TGF $\beta$-induced fibroblast-to-myofibroblast differentiation is associated with induction of NOX4 and thus presumably generation of $\mathrm{H}_{2} \mathrm{O}_{2}[84,85]$, which unlike superoxide does not appear to react directly with NO. Thus, any opposing regulation of myofibroblast differentiation by $\mathrm{NO} / \mathrm{cGMP}$ signaling and NOX4-derived $\mathrm{H}_{2} \mathrm{O}_{2}$ presumably occurs via distinct mechanism(s).

Several examples of opposing interaction between $\mathrm{H}_{2} \mathrm{O}_{2}$ and NO/cGMP signaling have been reported. For example, incubation of isolated rat hepatocytes with an $\mathrm{NO}$ donor prevented $\mathrm{H}_{2} \mathrm{O}_{2}$-induced cell death, presumably via cGMPactivated downstream signaling since the prosurvival effect of $\mathrm{NO}$ was ablated by cycloheximide indicating the requirement of de novo protein synthesis [135]. Conversely, $\mathrm{H}_{2} \mathrm{O}_{2}$ impaired NO production in porcine aortic endothelial cells, possibly via direct oxidative inactivation of eNOS cofactors [136]. Moreover, $\mathrm{H}_{2} \mathrm{O}_{2}$ eliminates the endotheliumdependent vasodilatory response to acetylcholine, a potent inducer of NO synthesis [137].

There are several potential mechanisms by which NOX4derived $\mathrm{H}_{2} \mathrm{O}_{2}$ and $\mathrm{NO} / \mathrm{cGMP}$ signaling may interact to elicit opposing functions during myofibroblast differentiation. For example, both NOS and NOX require NADPH as an electron donor for enzyme activity. NOX4 induction is an early event during TGF $\beta 1$-mediated differentiation $[3,20]$, whereas enhancing cGMP levels inhibits/reverses prostatic fibroblastto-myofibroblast differentiation without impairing NOX4 mRNA induction by TGF $\beta 1$ (our unpublished observations) [138], indicating that NO/cGMP signaling acts downstream of NOX4-derived $\mathrm{H}_{2} \mathrm{O}_{2}$ production. Thus, NADPH consumption/depletion due to elevated NOX4 activity may attenuate NO-dependent cGMP production and thereby impair NO/cGMP-mediated inhibition of differentiation.

A further potential mode of opposing interaction may occur via mutually exclusive modification of NOX/NO target proteins. For example, NO upregulates the activity of sarco/endoplasmic reticulum Ca2+ ATPase (SERCA) via $S$ glutathiolation on cysteine 674 [139]. Interestingly, TGF $\beta 1-$ mediated induction of NOX4 in aortic SMCs resulted in SERCA oxidation of the same thiol group, inhibited NOmediated $S$-glutathiolation, and attenuated NO inhibition of SMC migration [140,141]. Similar effects were observed upon exposure of vascular SMCs to $\mathrm{H}_{2} \mathrm{O}_{2}$ or high glucose, which induced NOX4 levels leading to SERCA oxidation, inhibition of NO-induced S-glutathiolation and migration. Moreover, NOX4 knockdown decreased SERCA oxidation and restored the inhibition of SMC migration by NO [142].

NOX4-derived $\mathrm{H}_{2} \mathrm{O}_{2}$ may also attenuate $\mathrm{NO}$ signaling by downregulating sGC expression/activity and consequently NO-dependent cGMP generation. $\mathrm{H}_{2} \mathrm{O}_{2}$ decreased sGC expression and NO-dependent cGMP generation in pulmonary arterial SMCs from lambs with persistent pulmonary hypertension of the newborn [143]. Moreover, incubation of rat aortic SMCs or freshly isolated vessels with ROSgenerating agents (including $\mathrm{H}_{2} \mathrm{O}_{2}$ ) significantly decreased sGC expression and reduced SNP-induced cGMP formation in the SMCs [144]. In addition, PTP inhibitors or $\mathrm{H}_{2} \mathrm{O}_{2}$ promoted tyrosine phosphorylation of the beta 1 subunit of sGC, most likely via Src-like kinases [145]. Subsequent studies revealed that cGMP levels are cross-regulated via a mechanism that involves c-Src-dependent phosphorylation of sGC, which attenuates sGC activity and cGMP formation [146]. These studies suggest that elevated NOX4-derived $\mathrm{H}_{2} \mathrm{O}_{2}$ during myofibroblast differentiation may oxidatively inactivate protein tyrosine phosphatases and/or activate Src kinase, which in turn promote inhibitory phosphorylation of sGC leading to reduced cGMP signaling.

Interactions between TGF $\beta$ and NO signaling have also been reported. For example, the NO donor $S$-nitroso$N$-acetyl-penicillamine or treatment with 8-bromo-cGMP decreased TGF $\beta 3$ mRNA levels in neonatal rat cardiac fibroblasts, whereas TGF $\beta 1 \mathrm{mRNA}$ levels were modestly increased [147]. Furthermore, $S$-nitroso- $N$-acetyl-penicillamine and ROS, most likely $\mathrm{H}_{2} \mathrm{O}_{2}$, both increased TGF $\beta 1$ release from human epithelial alveolar cells [148]. On the other hand, TGF $\beta 1$ decreased sGC and PKGI expression in pulmonary artery and aortic SMCs from adult rats and mice and a TGF $\beta$ neutralizing antibody prevented the reduction of sGC and PKGI protein expression in chronic oxygen-induced lung injury in mouse pups [149].

Thus, similar to reciprocal inhibition of superoxide and $\mathrm{NO}, \mathrm{H}_{2} \mathrm{O}_{2}$, and $\mathrm{NO}$ also appear to interact in a functionally opposing manner during myofibroblast differentiation. However, rather than direct radical quenching as observed for superoxide and NO, the basis of reciprocal inhibition between $\mathrm{H}_{2} \mathrm{O}_{2}$ and $\mathrm{NO}$ apparently occurs at multiple indirect levels. Cumulatively, such interplay would be expected to lead to downregulation of NO/cGMP signaling upon TGF $\beta 1$ mediated induction of NOX4-derived $\mathrm{H}_{2} \mathrm{O}_{2}$ and thereby promote fibroblast-to-myofibroblast differentiation. Consistent with this hypothesis, TGF $\beta 1$ significantly decreased NO production in dermal fibroblasts, whereas increasing NO signaling by stimulating sGC generation and/or inhibiting cGMP degradation counteracted ROS-mediated inactivation of NO signaling and prevented myofibroblast differentiation [122]. Inhibition of differentiation can also be achieved by treatment with 8 -bromo-cGMP [122], indicating that suppression of myofibroblast differentiation by enhanced NO signaling is mediated by downstream cGMP-dependent mechanisms and not via the NO radical per se.

\section{Reversal of Myofibroblast Differentiation and Clinical Implications}

Tissue fibrosis is thought to arise from failure of the myofibroblast apoptotic wave during wound healing [12, 45]. Thus, whilst inhibition of myofibroblast differentiation may be suitable to prevent disease progression, curative treatments would also require targeting of preexisting myofibroblasts. Although some promising data for such approaches exist [150], extensive fibroblast heterogeneity and the lack of a consensus "myofibroblast-specific" surface marker mean that tissue- and even disease-specific targeting strategies will be required.

An alternative approach to clear the fibrotic myofibroblast pool envisages inducing their dedifferentiation to the 


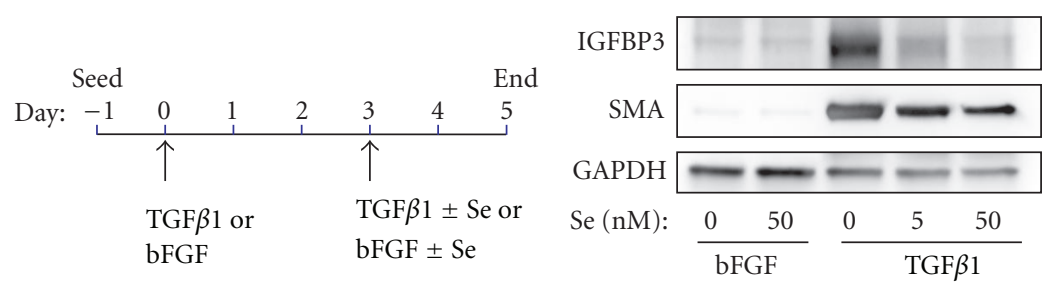

(a)

(b)
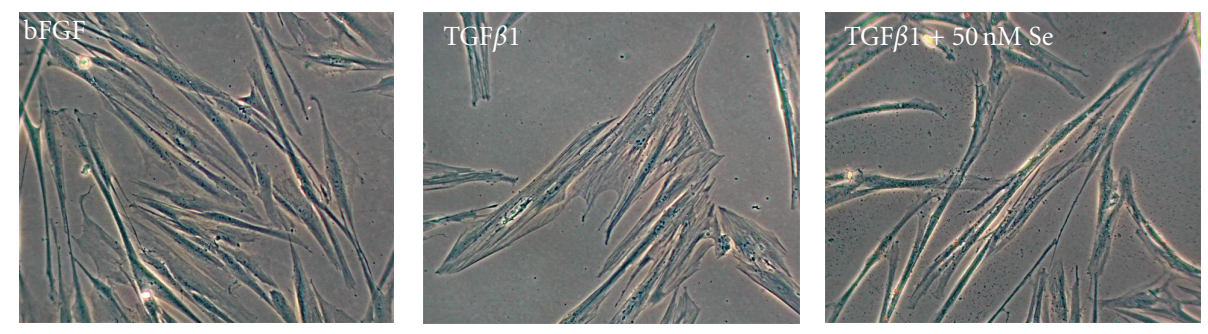

(c)

FIGURE 2: Selenium reverses myofibroblast differentiation of prostatic fibroblasts. Therapeutic targeting of myofibroblast dysregulation in fibrotic disease may be accomplished by promoting myofibroblast dedifferentiation to the nonactivated fibroblast/progenitor phenotype. (a) Methodology outline of selenium (Se)-mediated reversal of fibroblast-to-myofibroblast differentiation in primary human prostatic fibroblasts. Myofibroblast differentiation was induced with $1 \mathrm{ng} / \mathrm{mL}$ TGF $\beta 1$ or bFGF as mock control. After 72 hours, fresh media was added containing bFGF or TGF $\beta 1$ as before but supplemented with selenium (Se) as sodium selenite or vehicle equivalent. Cells were incubated for a further 48 hours before harvesting. Reversal of myofibroblast differentiation of primary human prostatic fibroblasts treated as outlined in (a) was verified by (b) Western blotting for the myofibroblast markers, $\alpha$-SMA, and IGFBP3 and (c) morphological analysis using phase contrast microscopy. (b) Induction of myofibroblast differentiation by TGF $\beta 1$ in the absence of Se $(0 \mathrm{nM})$ is indicated by increased production of myofibroblast markers. However, both $\alpha$-SMA and IGFBP3 levels are reduced in the presence of Se in a dose-dependent manner. (c) At the morphological level, Se restores the thin, elongated and light refractive phenotype to cells predifferentiated with TGF $\beta 1$ (far right), whereas cells treated with TGF $\beta 1$ alone (center panel) exhibit the typical enlarged and flattened myofibroblast phenotype with visible actin-like filaments. (b-c) Images are representative of three independent experiments using primary cells isolated from different donors.

nonactivated fibroblast/progenitor phenotype. It has long been considered that fibrosis and fibroblast-to-myofibroblast differentiation are irreversible processes. However, recent data from in vitro and in vivo models indicate that tissue fibrosis and fibroblast-to-myofibroblast differentiation can indeed be reversed. For example, we observe that exogenous Se or PDE5 inhibition restore morphological and molecular characteristics typical of the fibroblast phenotype to in vitro differentiated prostatic myofibroblasts even in the continued presence of the TGF $\beta$ differentiation-inducing stimulus (Figure 2) [138]. Our data are supported by studies employing myofibroblasts from IPF patients and a threedimensional coculture model of porcine skin fibrosis that similarly demonstrate the potential utility of ROS scavenging in promoting myofibroblast dedifferentiation [151, 152]. Moreover, treatment of in vitro differentiated corneal myofibroblasts with fibroblast growth factor in combination with heparin decreased expression of $\alpha$-SMA, TGF $\beta$ receptors, and cadherins, indicating reversal of myofibroblast differentiation to a fibroblast-like phenotype [153]. Recently, pharmacological inhibition of NOX4 after induction of liver fibrosis in mice was shown to reduce ROS levels and significantly attenuate fibrosis [22].

The principle of enhancing NO/cGMP signaling to induce myofibroblast to fibroblast reversal has also been successfully demonstrated in animal models. Treatment with the PDE5 inhibitor vardenafil reduced myofibroblast numbers and total size of preformed TGF $\beta 1$-induced Peyronie's disease plaques in rats [154]. Moreover, BAY 41-2272 reduced established fibrosis in a modified mouse model of bleomycin-induced skin fibrosis and in Tsk-1 mice [124].

Collectively, these findings and those discussed above suggest that signaling via ROS and local growth factors (such as TGF $\beta$ and fibroblast growth factor) play key roles not only in driving fibroblast-to-myofibroblast differentiation but also in subsequently maintaining the myofibroblast phenotype. Thus, we hypothesize that high NO/cGMP signaling and low NOX4-derived ROS production coordinately maintain the fibroblast phenotype, whereas differentiation into the myofibroblast phenotype may proceed upon elevated NOX4-derived ROS signaling and/or concomitant inactivation of NO/cGMP signaling (Figure 3).

The observation that fibroblasts and myofibroblasts are interconvertible phenotypes rather than terminally differentiated cell types has significant clinical implications for potential curative therapy of advanced fibrotic disease. In this respect, pharmacological regulation of redox signaling via NOX4 inhibitors, antioxidants, and/or enhancement of NO/cGMP signaling provides a promising option to modulate the fibroblast/myofibroblast ratio at multiple levels in several pathological conditions (Figure 3). For example, local activation of myofibroblast differentiation may provide a means to overcome impaired wound healing in the elderly, 


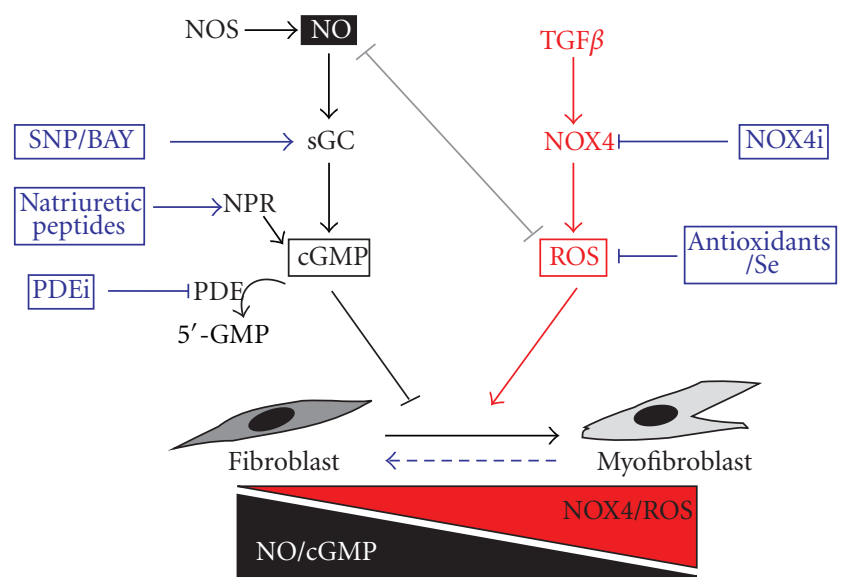

FIGURE 3: Potential therapeutic targeting of myofibroblast differentiation in the treatment of age-associated fibrotic disease. Myofibroblast differentiation induced by TGF $\beta 1$ plays a central role in the etiology of numerous age-related fibrotic disorders. In particular, TGF $\beta$ induces a prooxidant shift in intracellular redox homeostasis via the induction of NOX4-derived ROS (in particular $\mathrm{H}_{2} \mathrm{O}_{2}$ ), which modulates downstream phosphorylation signaling cascades and transcriptional events that culminate in myofibroblast differentiation. The concomitant downregulation of selenium-dependent ROS scavenging enzymes by TGF $\beta$ further potentiates NOX4-derived ROS signaling, which also downregulates NO/cGMP signaling and thereby relieves inhibition of myofibroblast differentiation by NO. Fibroblast-to-myofibroblast differentiation and subsequent tissue fibrosis are reversible processes (broken horizontal arrow). Thus, pharmacological interference of these redox signaling processes to redress redox homeostasis and thereby restore the physiological fibroblast: myofibroblast ratio offers a promising therapeutic strategy for the treatment of age-related pathologies associated with myofibroblast dysregulation. Such pharmacological targeting may succeed at multiple levels. For example, targeting NOX4 directly via NOX4 inhibitors (NOX4i) or indirectly by ROS scavenging with Se or antioxidants would attenuate NOX4-derived ROS signaling and restore the inhibitory effects on differentiation by NO/cGMP signaling. Enhancing NO/cGMP signaling is also sufficient to prevent and reverse fibroblast-to-myofibroblast differentiation and may be achieved by (i) sGC stimulation using the NO donor sodium nitroprusside (SNP) and/or direct sGC stimulators/activators (BAY), (ii) promoting cGMP synthesis via administration of natriuretic peptides that bind to and activate transmembrane guanylyl cyclase natriuretic peptide receptors (NPRs), or (iii) by inhibiting phosphodiesterase (PDE)-mediated cGMP hydrolysis using PDE inhibitors (PDEi).

whereas inhibiting fibroblast-to-myofibroblast differentiation and restoring the fibroblast phenotype may represent a therapeutic strategy for fibrotic disease and also serve as a stromal-targeted chemotherapy approach for solid tumors, such as breast, liver, and prostate cancer.

Despite its critical role in the pathogenesis of fibrosis, $\operatorname{TGF} \beta$ is not considered a direct clinical target due to its critical function in diverse biological processes and homeostatic maintenance [155]. As central downstream components of TGF $\beta$ signaling during fibroblast-to-myofibroblast differentiation, targeting NOX4-derived ROS and NO/cGMP might also be expected to elicit broad and undesirable effects. However, PDE5 inhibitors have a successful history in the treatment of ED, pulmonary arterial hypertension, and $\mathrm{BPH}$ [114-116]. Moreover, it should be noted that (i) none of the published Nox4 knockout animals display an obvious basal phenotype, (ii) a dual NOX1/NOX4 inhibitor (at doses that reduced liver fibrosis in mice) does not exert toxic effects in animal models, and (iii) the same inhibitor was also well tolerated in phase I clinical trials $[22,81]$. This likely reflects observations that NOX4 does not mediate all of the signaling functions of TGF $\beta$, as illustrated by the delayed temporal induction of NOX4 expression and ROS production $(\sim 2$ hours following TGF $\beta 1$ treatment) compared to the rapid induction of phosphorylation cascades, such as phosphorylation of Smad2/3 and ERK1/2 that occurs within 5 minutes of TGF $\beta$ treatment $[3,20,83]$. Thus, selective modulation of NOX4, PDE5, and/or sGC activities may permit continued physiological ROS/NO signaling due to the presence of multiple NOX, PDE, and GC isoforms. Moreover, the fact that these enzymes belong to multimembered families may be clinically exploited to selectively target those isoforms underlying pathology in a given tissue or disease state. For example in the heart, PDE1A appears to play a critical role in cardiac fibrosis and its selective targeting in rats and mice led to regression of cardiac remodeling that is associated with various cardiac diseases [156].

\section{Conclusions}

Myofibroblast activation and differentiation are central processes of normal wound healing. However, these beneficial effects of myofibroblasts are dysregulated in fibrotic disorders and in the reactive stromal response that promotes tumorigenesis and metastasis. Whilst TGF $\beta$ plays a key role in initiating myofibroblast differentiation and ECM deposition during normal wound healing, failure of myofibroblast clearance together with their autocrine production of TGF $\beta$ leads to ECM accumulation, fibrosis, and ultimately organ dysfunction. A large body of data from in vitro fibroblast-to-myofibroblast differentiation models indicates that TGF $\beta 1$-induced myofibroblast differentiation is mediated via induction of NOX4-derived $\mathrm{H}_{2} \mathrm{O}_{2}$, which 
modulates downstream phosphorylation signaling cascades and transcriptional events that culminate in cytoskeletal remodeling and myofibroblast differentiation. In addition, NOX4-derived $\mathrm{H}_{2} \mathrm{O}_{2}$ appears to downregulate NO/cGMP signaling via multiple mechanisms and thereby relieves inhibition of myofibroblast differentiation by NO. Findings from these in vitro cellular models of fibrosis are supported by extensive in vivo data, underscoring the value of in vitro models in defining molecular mechanisms underlying fibrogenic disease and serving as screening platforms for the discovery of novel therapeutics. Targeting NOX4 directly via NOX4 inhibitors or indirectly by ROS scavenging with Se, antioxidants or enhancing NO/cGMP signaling by sGC stimulation, and/or inhibition of cGMP degradation attenuates TGF $\beta 1$-induced differentiation and inhibits myofibroblast activation. Moreover, these agents induce the dedifferentiation/reversal of preexisting myofibroblasts to a quiescent fibroblast phenotype. Thus, pharmacological interference of these redox signaling processes to restore the physiological fibroblast: myofibroblast ratio offers a promising therapeutic strategy not only for the treatment of fibrotic diseases but also for managing tumor invasion and metastasis at the level of stromal remodeling.

\section{Abbreviations}

$\alpha$-SMA: Alpha smooth muscle cell actin

bFGF: Basic fibroblast growth factor

BPH: Benign prostatic hyperplasia

cAMP: Cyclic adenosine monophosphate

cGMP: Cyclic guanosine monophosphate

ECM: $\quad$ Extracellular matrix

ED: $\quad$ Erectile dysfunction

IPF: Idiopathic pulmonary fibrosis

L-NAME: $N_{\omega}$-nitro-L-arginine methyl ester

LUTS: Lower urinary tract symptoms

MAPK: Mitogen-associated protein kinase

NO: $\quad$ Nitric oxide

NOS: $\quad$ Nitric oxide synthase

NOX: NADPH oxidase

PDE: $\quad$ Phosphodiesterase

PKG: $\quad$ Protein kinase G

PTK: $\quad$ Protein tyrosine kinase

PTP: $\quad$ Protein tyrosine phosphatase

RNS: $\quad$ Reactive nitrogen species

ROS: Reactive oxygen species

Se: Selenium

sGC: $\quad$ Soluble guanylyl cyclase

SMC: $\quad$ Smooth muscle cell

SNP: $\quad$ Sodium nitroprusside

TGF $\beta$ : Transforming growth factor beta.

\section{Acknowledgments}

N. Sampson is supported by an Elise Richter postdoctoral fellowship from the Austrian Science Fund (FWF; V216B13). P. Berger is supported by the Austrian Science Fund (FWF; NRN S9307-B05).

\section{References}

[1] B. Hinz, S. H. Phan, V. J. Thannickal et al., "Recent developments in myofibroblast biology: paradigms for connective tissue remodeling," The American Journal of Pathology, vol. 180, no. 4, pp. 1340-1355, 2012.

[2] G. Gabbiani, "The myofibroblast in wound healing and fibrocontractive diseases," Journal of Pathology, vol. 200, no. 4, pp. 500-503, 2003.

[3] N. Sampson, R. Koziel, C. Zenzmaier et al., "ROS signaling by NOX4 drives fibroblast-to-myofibroblast differentiation in the diseased prostatic stroma," Molecular Endocrinology, vol. 25, no. 3, pp. 503-515, 2011.

[4] A. T. Grazul-Bilska, M. L. Johnson, J. J. Bilski et al., "Wound healing: the role of growth factors," Drugs of Today, vol. 39, no. 10, pp. 787-800, 2003.

[5] I. Haase, R. Evans, R. Pofahl, and F. M. Watt, "Regulation of keratinocyte shape, migration and wound epithelialization by IGF-1 and EGF-dependent signalling pathways," Journal of Cell Science, vol. 116, no. 15, pp. 3227-3238, 2003.

[6] A. Desmouliere, M. Redard, I. Darby, and G. Gabbiani, "Apoptosis mediates the decrease in cellularity during the transition between granulation tissue and scar," American Journal of Pathology, vol. 146, no. 1, pp. 56-66, 1995.

[7] J. I. Jun and L. F. Lau, "Cellular senescence controls fibrosis in wound healing," Aging, vol. 2, no. 9, pp. 627-631, 2010.

[8] G. N. Pitiyage, P. Slijepcevic, A. Gabrani et al., "Senescent mesenchymal cells accumulate in human fibrosis by a telomere-independent mechanism and ameliorate fibrosis through matrix metalloproteinases," Journal of Pathology, vol. 223, no. 5, pp. 604-617, 2011.

[9] S. L. Schissel and M. D. Layne, "Telomerase, myofibroblasts, and pulmonary fibrosis," American Journal of Respiratory Cell and Molecular Biology, vol. 34, no. 5, pp. 520-522, 2006.

[10] P. D. Adams, "Healing and hurting: molecular mechanisms, functions, and pathologies of cellular senescence," Molecular Cell, vol. 36, no. 1, pp. 2-14, 2009.

[11] A. Gosain and L. A. DiPietro, "Aging and wound healing," World Journal of Surgery, vol. 28, no. 3, pp. 321-326, 2004.

[12] K. Kis, X. Liu, and J. S. Hagood, "Myofibroblast differentiation and survival in fibrotic disease," Expert Reviews in Molecular Medicine, vol. 13, article e27, 2011.

[13] S. Ueha, F. H. Shand, and K. Matsushima, "Cellular and molecular mechanisms of chronic inflammation-associated organ fibrosis," Frontiers in Immunology, vol. 3, p. 71, 2012.

[14] M. Amano, M. Nakayama, and K. Kaibuchi, "Rho-kinase/ ROCK: a key regulator of the cytoskeleton and cell polarity," Cytoskeleton, vol. 67, no. 9, pp. 545-554, 2010.

[15] J. J. Tomasek, G. Gabbiani, B. Hinz, C. Chaponnier, and R. A. Brown, "Myofibroblasts and mechano: regulation of connective tissue remodelling," Nature Reviews Molecular Cell Biology, vol. 3, no. 5, pp. 349-363, 2002.

[16] Y. Kojima, A. Acar, E. N. Eaton et al., "Autocrine TGF- $\beta$ and stromal cell-derived factor-1 (SDF-1) signaling drives the evolution of tumor-promoting mammary stromal myofibroblasts," Proceedings of the National Academy of Sciences of the United States of America, vol. 107, no. 46, pp. 2000920014, 2010.

[17] L. Ronnov-Jessen and O. W. Petersen, "Induction of $\alpha$ smooth muscle actin by transforming growth factor- $\beta 1$ in quiescent human breast gland fibroblasts," Laboratory Investigation, vol. 68, no. 6, pp. 696-707, 1993.

[18] A. Desmouliere, A. Geinoz, F. Gabbiani et al., "Transforming growth factor- $\beta 1$ induces $\alpha$-smooth muscle actin expression 
in granulation tissue myofibroblasts and in quiescent and growing cultured fibroblasts," Journal of Cell Biology, vol. 122, no. 1, pp. 103-111, 1993.

[19] D. M. Peehl and R. G. Sellers, "Induction of smooth muscle cell phenotype in cultured human prostatic stromal cells," Experimental Cell Research, vol. 232, no. 2, pp. 208-215, 1997.

[20] I. Cucoranu, R. Clempus, A. Dikalova et al., "NAD(P)H oxidase 4 mediates transforming growth factor- $\beta 1$-induced differentiation of cardiac fibroblasts into myofibroblasts," Circulation Research, vol. 97, no. 9, pp. 900-907, 2005.

[21] C. D. Bondi, N. Manickam, D. Y. Lee et al., "NAD(P)H oxidase mediates TGF- $\beta 1$-induced activation of kidney myofibroblasts," Journal of the American Society of Nephrology, vol. 21, no. 1, pp. 93-102, 2010.

[22] J. X. Jiang, X. Chen, N. Serizawa et al., "Liver fibrosis and hepatocyte apoptosis are attenuated by GKT137831, a novel NOX4/NOX1 inhibitor in vivo," Free Radical Biology \& Medicine, vol. 53, no. 2, pp. 289-296, 2012.

[23] T. R. Dunkern, D. Feurstein, G. A. Rossi, F. Sabatini, and A. Hatzelmann, "Inhibition of TGF- $\beta$ induced lung fibroblast to myofibroblast conversion by phosphodiesterase inhibiting drugs and activators of soluble guanylyl cyclase," European Journal of Pharmacology, vol. 572, no. 1, pp. 12-22, 2007.

[24] A. Biernacka, M. Dobaczewski, and N. G. Frangogiannis, "TGF-beta signaling in fibrosis," Growth Factors, vol. 29, no. 5, pp. 196-202, 2011.

[25] G. Untergasser, R. Gander, C. Lilg, G. Lepperdinger, E. Plas, and P. Berger, "Profiling molecular targets of TGF- $\beta 1$ in prostate fibroblast-to- myofibroblast transdifferentiation," Mechanisms of Ageing and Development, vol. 126, no. 1, pp. 59-69, 2005.

[26] J. Folkman, M. Klagsbrun, J. Sasse, M. Wadzinski, D. Ingber, and I. Vlodavsky, "A heparin-binding angiogenic proteinbasic fibroblast growth factor-is stored within basement membrane," American Journal of Pathology, vol. 130, no. 2, pp. 393-400, 1988.

[27] G. S. Schultz and A. Wysocki, "Interactions between extracellular matrix and growth factors in wound healing," Wound Repair and Regeneration, vol. 17, no. 2, pp. 153-162, 2009.

[28] D. R. Senger, K. P. Claffey, J. E. Benes, C. A. Perruzzi, A. P. Sergiou, and M. Detmar, "Angiogenesis promoted by vascular endothelial growth factor: regulation through $\alpha 1 \beta 1$ and $\alpha 2 \beta 1$ integrins," Proceedings of the National Academy of Sciences of the United States of America, vol. 94, no. 25, pp. 13612-13617, 1997.

[29] J. Rosenbloom, S. V. Castro, and S. A. Jimenez, "Narrative review: fibrotic diseases: cellular and molecular mechanisms and novel therapies," Annals of Internal Medicine, vol. 152, no. 3, pp. 159-166, 2010.

[30] K. M. Mak, A. J. Kwong, E. Chu et al., "Hepatic steatosis, fibrosis, and cancer in elderly cadavers," The Anatomical Record (Hoboken), vol. 295, no. 1, pp. 40-50, 2012.

[31] J. T. Isaacs, "Etiology of benign prostatic hyperplasia," European Urology, vol. 25, no. 1, pp. 6-9, 1994.

[32] N. Sampson, G. Untergasser, E. Plas, and P. Berger, "The ageing male reproductive tract," Journal of Pathology, vol. 211, no. 2, pp. 206-218, 2007.

[33] G. Pannarale, R. Carbone, G. Del Mastro et al., "The aging kidney: structural changes," Journal of Nephrology, vol. 23, no. 15, pp. S37-S40, 2010.

[34] M. Volkova, Y. Zhang, A. C. Shaw et al., "The role of Tolllike receptors in age-associated lung diseases," The Journals of Gerontology A, vol. 67, no. 3, pp. 247-253, 2012.
[35] D. F. Dai, T. Chen, S. C. Johnson et al., "Cardiac aging: from molecular mechanisms to significance in human health and disease," Antioxidants \& Redox Signaling, vol. 16, no. 12, pp. 1492-1526, 2012.

[36] H. Nakagawa and S. Maeda, "Molecular mechanisms of liver injury and hepatocarcinogenesis: focusing on the role of stress-activated MAPK," Pathology Research International, vol. 2012, Article ID 172894, 2012.

[37] K. S. Sfanos and A. M. De Marzo, "Prostate cancer and inflammation: the evidence," Histopathology, vol. 60, no. 1, pp. 199-215, 2012.

[38] M. Otranto, V. Sarrazy, F. Bonte et al., "The role of the myofibroblast in tumor stroma remodeling," Cell Adhesion \& Migration, vol. 6, no. 3, 2012.

[39] J. A. Tuxhorn, G. E. Ayala, M. J. Smith, V. C. Smith, T. D. Dang, and D. R. Rowley, "Reactive stroma in human prostate cancer: induction of myofibroblast phenotype and extracellular matrix remodeling," Clinical Cancer Research, vol. 8, no. 9, pp. 2912-2923, 2002.

[40] M. Ao, O. E. Franco, D. Park, D. Raman, K. Williams, and S. W. Hayward, "Cross-talk between paracrine-acting cytokine and chemokine pathways promotes malignancy in benign human prostatic epithelium," Cancer Research, vol. 67, no. 9, pp. 4244-4253, 2007.

[41] F. Yang, J. A. Tuxhorn, S. J. Ressler, S. J. McAlhany, T. D. Dang, and D. R. Rowley, "Stromal expression of connective tissue growth factor promotes angiogenesis and prostate cancer tumorigenesis," Cancer Research, vol. 65, no. 19, pp. 88878895, 2005.

[42] J. A. Tuxhorn, S. J. McAlhany, F. Yang, T. D. Dang, and D. R. Rowley, "Inhibition of transforming growth factor- $\beta$ activity decreases angiogenesis in a human prostate cancer-reactive stroma xenograft model," Cancer Research, vol. 62, no. 21, pp. 6021-6025, 2002.

[43] P. Lu, K. Takai, V. M. Weaver et al., "Extracellular matrix degradation and remodeling in development and disease," Cold Spring Harbor Perspectives in Biology, vol. 3, no. 12, 2011.

[44] H. Ikushima and K. Miyazono, "TGF $\beta 2$ signalling: a complex web in cancer progression," Nature Reviews Cancer, vol. 10, no. 6, pp. 415-424, 2010.

[45] S. Chabaud, M. P. Corriveau, T. Grodzicky et al., "Decreased secretion of MMP by non-lesional late-stage scleroderma fibroblasts after selection via activation of the apoptotic faspathway," Journal of Cellular Physiology, vol. 226, no. 7, pp. 1907-1914, 2011.

[46] C. K. Garcia, "Idiopathic pulmonary fibrosis: update on genetic discoveries," Proceedings of the American Thoracic Society, vol. 8, no. 2, pp. 158-162, 2011.

[47] J. T. Cronkhite, C. Xing, G. Raghu et al., "Telomere shortening in familial and sporadic pulmonary fibrosis," American Journal of Respiratory and Critical Care Medicine, vol. 178, no. 7, pp. 729-737, 2008.

[48] A. L. Degryse, X. C. Xu, J. L. Newman et al., "Telomerase deficiency does not alter bleomycin-induced fibrosis in mice," Experimental Lung Research, vol. 38, no. 3, pp. 124-134, 2012.

[49] J. C. Horowitz, D. S. Rogers, V. Sharma et al., "Combinatorial activation of FAK and AKT by transforming growth factor$\beta 1$ confers an anoikis-resistant phenotype to myofibroblasts," Cellular Signalling, vol. 19, no. 4, pp. 761-771, 2007.

[50] P. Kulasekaran, C. A. Scavone, D. S. Rogers, D. A. Arenberg, V. J. Thannickal, and J. C. Horowitz, "Endothelin-1 and transforming growth factor- $\beta 1$ independently induce fibroblast resistance to apoptosis via AKT activation," American Journal 
of Respiratory Cell and Molecular Biology, vol. 41, no. 4, pp. 484-493, 2009.

[51] B. Hu, D. C. Tack, T. Liu, Z. Wu, M. R. Ullenbruch, and S. H. Phan, "Role of Smad3 in the regulation of rat telomerase reverse transcriptase by TGF $\beta$," Oncogene, vol. 25, no. 7, pp. 1030-1041, 2006.

[52] H. Li, D. Xu, J. Li, M. C. Berndt, and J. P. Liu, “Transforming growth factor $\beta$ suppresses human telomerase reverse transcriptase (hTERT) by Smad3 interactions with c-Myc and the hTERT gene," Journal of Biological Chemistry, vol. 281, no. 35, pp. 25588-25600, 2006.

[53] C. Beyer, G. Schett, O. Distler, and J. H. W. Distler, "Animal models of systemic sclerosis: prospects and limitations," Arthritis and Rheumatism, vol. 62, no. 10, pp. 2831-2844, 2010.

[54] J. Gauldie and M. Kolb, "Animal models of pulmonary fibrosis: how far from effective reality?" American Journal of Physiology, vol. 294, no. 2, p. L151, 2008.

[55] B. B. Moore and C. M. Hogaboam, "Murine models of pulmonary fibrosis," American Journal of Physiology, vol. 294, no. 2, pp. L152-L160, 2008.

[56] Y. Sun and K. T. Weber, "Animal models of cardiac fibrosis," Methods in Molecular Medicine, vol. 117, pp. 273-290, 2005.

[57] C. Weiler-Normann, J. Herkel, and A. W. Lohse, "Mouse models of liver fibrosis," Zeitschrift fur Gastroenterologie, vol. 45, no. 1, pp. 43-50, 2007.

[58] M. Zeisberg, M. A. Soubasakos, and R. Kalluri, "Animal models of renal fibrosis," Methods in Molecular Medicine, vol. 117, pp. 261-272, 2005.

[59] P. Roy-Burman, H. Wu, W. C. Powell, J. Hagenkord, and M. B. Cohen, "Genetically defined mouse models that mimic natural aspects of human prostate cancer development," Endocrine-Related Cancer, vol. 11, no. 2, pp. 225-254, 2004.

[60] P. Sharma and N. Schreiber-Agus, "Mouse models of prostate cancer," Oncogene, vol. 18, no. 38, pp. 5349-5355, 1999.

[61] J. P. Mizgerd and S. J. Skerrett, "Animal models of human pneumonia," American Journal of Physiology, vol. 294, no. 3, pp. L387-L398, 2008.

[62] G. Rowden, "Macrophages and dendritic cells in the skin. The skin immune system (SIS)," in Cutaneous Immunology and Clinical Immunodermatology, J. D. Bos, Ed., pp. 104-146, CRC Press, New York, NY, USA, 1997.

[63] E. Bell, B. Ivarsson, and C. Merrill, "Production of a tissuelike structure by contraction of collagen lattices by human fibroblasts of different proliferative potential in vitro," Proceedings of the National Academy of Sciences of the United States of America, vol. 76, no. 3, pp. 1274-1278, 1979.

[64] R. H. Cunnington, B. Wang, S. Ghavami, K. L. Bathe, S. G. Rattan, and I. M. C. Dixon, "Antifibrotic properties of c-Ski and its regulation of cardiac myofibroblast phenotype and contractility," American Journal of Physiology, vol. 300, no. 1, pp. C176-C186, 2011.

[65] T. Kohyama, X. Liu, F. Q. Wen et al., "PDE4 inhibitors attenuate fibroblast chemotaxis and contraction of native collagen gels," American Journal of Respiratory Cell and Molecular Biology, vol. 26, no. 6, pp. 694-701, 2002.

[66] H. Pan, J. Chen, J. Xu, M. Chen, and R. Ma, "Antifibrotic effect by activation of peroxisome proliferator-activated receptor-gamma in corneal fibroblasts.," Molecular Vision, vol. 15 , pp. 2279-2286, 2009.

[67] Q. Shi, X. Liu, Y. Bai et al., "In vitro effects of pirfenidone on cardiac fibroblasts: proliferation, myofibroblast differentiation, migration and cytokine secretion," PLoS ONE, vol. 6, no. 11, article e28134, 2011.
[68] C. Y. Tsai, K. I. Hata, S. Torii, M. Matsuyama, and M. Ueda, "Contraction potency of hypertrophic scar-derived fibroblasts in a connective tissue model: in vitro analysis of wound contraction," Annals of Plastic Surgery, vol. 35, no. 6, pp. 638-646, 1995.

[69] F. Q. Wen, C. M. Sköld, X. D. Liu et al., "Glucocorticoids and TGF- $\beta 1$ synergize in augmenting fibroblast mediated contraction of collagen gels," Inflammation, vol. 25, no. 2, pp. 109-117, 2001

[70] M. Yokozeki, K. Moriyama, H. Shimokawa, and T. Kuroda, "Transforming growth factor- $\beta 1$ modulates myofibroblastic phenotype of rat palatal fibroblasts in Vitro," Experimental Cell Research, vol. 231, no. 2, pp. 328-336, 1997.

[71] H. Y. Zhang, M. Gharaee-Kermani, K. Zhang, S. Karmiol, and S. H. Phan, "Lung fibroblast $\alpha$-smooth muscle actin expression and contractile phenotype in bleomycin-induced pulmonary fibrosis," American Journal of Pathology, vol. 148, no. 2, pp. 527-537, 1996.

[72] H. Rumpold, K. Mascher, G. Untergasser, E. Plas, M. Hermann, and P. Berger, "Trans-differentiation of prostatic stromal cells leads to decreased glycoprotein hormone $\alpha$ production," Journal of Clinical Endocrinology and Metabolism, vol. 87, no. 11, pp. 5297-5303, 2002.

[73] C. Zenzmaier, N. Sampson, D. Pernkopf, E. Plas, G. Untergasser, and P. Berger, "Attenuated proliferation and transdifferentiation of prostatic stromal cells indicate suitability of phosphodiesterase type 5 inhibitors for prevention and treatment of benign prostatic hyperplasia," Endocrinology, vol. 151, no. 8, pp. 3975-3984, 2010.

[74] L. Hecker, R. Vittal, T. Jones et al., "NADPH oxidase-4 mediates myofibroblast activation and fibrogenic responses to lung injury," Nature Medicine, vol. 15, no. 9, pp. 10771081, 2009.

[75] N. Amara, D. Goven, F. Prost, R. Muloway, B. Crestani, and J. Boczkowski, "NOX4/NADPH oxidase expression is increased in pulmonary fibroblasts from patients with idiopathic pulmonary fibrosis and mediates TGF $\beta 1$-induced fibroblast differentiation into myofibroblasts," Thorax, vol. 65, no. 8 , pp. 733-738, 2010.

[76] P. Li, D. Wang, J. Lucas et al., "Atrial natriuretic peptide inhibits transforming growth factor $\beta$-induced Smad signaling and myofibroblast transformation in mouse cardiac fibroblasts," Circulation Research, vol. 102, no. 2, pp. 185-192, 2008.

[77] H. Masuyama, T. Tsuruda, Y. Sekita et al., "Pressure-independent effects of pharmacological stimulation of soluble guanylate cyclase on fibrosis in pressure-overloaded rat heart," Hypertension Research, vol. 32, no. 7, pp. 597-603, 2009.

[78] D. Harman, "Aging: a theory based on free radical and radiation chemistry," Journal of Gerontology, vol. 11, no. 3, pp. 298-300, 1956.

[79] H. J. Forman, J. M. Fukuto, T. Miller, H. Zhang, A. Rinna, and S. Levy, "The chemistry of cell signaling by reactive oxygen and nitrogen species and 4-hydroxynonenal," Archives of Biochemistry and Biophysics, vol. 477, no. 2, pp. 183-195, 2008.

[80] F. Jiang, Y. Zhang, and G. J. Dusting, "NADPH oxidasemediated redox signaling: roles in cellular stress response, stress tolerance, and tissue repair," Pharmacological Reviews, vol. 63, no. 1, pp. 218-242, 2011.

[81] S. Altenhofer, P. W. Kleikers, K. A. Radermacher et al., "The NOX toolbox: validating the role of NADPH oxidases in physiology and disease," Cellular and Molecular Life Sciences, vol. 69, no. 14, pp. 2327-2343, 2012. 
[82] K. D. Martyn, L. M. Frederick, K. Von Loehneysen, M. C. Dinauer, and U. G. Knaus, "Functional analysis of NOX4 reveals unique characteristics compared to other NADPH oxidases," Cellular Signalling, vol. 18, no. 1, pp. 69-82, 2006.

[83] L. Serrander, L. Cartier, K. Bedard et al., "NOX4 activity is determined by mRNA levels and reveals a unique pattern of ROS generation,” Biochemical Journal, vol. 406, no. 1, pp. 105-114, 2007.

[84] S. I. Dikalov, A. E. Dikalova, A. T. Bikineyeva, H. H. H. W. Schmidt, D. G. Harrison, and K. K. Griendling, "Distinct roles of NOX1 and NOX4 in basal and angiotensin IIstimulated superoxide and hydrogen peroxide production," Free Radical Biology and Medicine, vol. 45, no. 9, pp. 13401351, 2008.

[85] I. Takac, K. Schröder, L. Zhang et al., "The E-loop is involved in hydrogen peroxide formation by the NADPH oxidase NOX4," Journal of Biological Chemistry, vol. 286, no. 15, pp. 13304-13313, 2011.

[86] K. Bedard and K. H. Krause, "The NOX family of ROSgenerating NADPH oxidases: physiology and pathophysiology," Physiological Reviews, vol. 87, no. 1, pp. 245-313, 2007.

[87] D. Trachootham, W. Lu, M. A. Ogasawara, N. R. D. Valle, and P. Huang, "Redox regulation of cell survival," Antioxidants and Redox Signaling, vol. 10, no. 8, pp. 1343-1374, 2008.

[88] J. L. Barnes and Y. Gorin, "Myofibroblast differentiation during fibrosis: role of $\mathrm{NAD}(\mathrm{P}) \mathrm{H}$ oxidases," Kidney International, vol. 79, no. 9, pp. 944-956, 2011.

[89] K. A. Graham, M. Kulawiec, K. M. Owens et al., "NADPH oxidase 4 is an oncoprotein localized to mitochondria," Cancer Biology and Therapy, vol. 10, no. 3, pp. 223-231, 2010.

[90] J. Kuroda, T. Ago, S. Matsushima, P. Zhai, M. D. Schneider, and J. Sadoshima, "NADPH oxidase 4 (NOX4) is a major source of oxidative stress in the failing heart," Proceedings of the National Academy of Sciences of the United States of America, vol. 107, no. 35, pp. 15565-15570, 2010.

[91] S. J. Guo, L. Y. Wu, W. L. Shen et al., "Gene profile for differentiation of vascular adventitial myofibroblasts," Sheng Li Xue Bao, vol. 58, no. 4, pp. 337-344, 2006.

[92] T. Hu, S. P. Ramachandrarao, S. Siva et al., "Reactive oxygen species production via NADPH oxidase mediates TGF- $\beta$ induced cytoskeletal alterations in endothelial cells," American Journal of Physiology, vol. 289, no. 4, pp. F816-F825, 2005.

[93] R. E. Clempus, D. Sorescu, A. E. Dikalova et al., "NOX4 is required for maintenance of the differentiated vascular smooth muscle cell phenotype," Arteriosclerosis, Thrombosis, and Vascular Biology, vol. 27, no. 1, pp. 42-48, 2007.

[94] K. Schröder, K. Wandzioch, I. Helmcke, and R. P. Brandes, "NOX4 acts as a switch between differentiation and proliferation in preadipocytes," Arteriosclerosis, Thrombosis, and Vascular Biology, vol. 29, no. 2, pp. 239-245, 2009.

[95] J. C. Pache, S. Carnesecchi, C. Deffert et al., "NOX-4 is expressed in thickened pulmonary arteries in idiopathic pulmonary fibrosis," Nature Medicine, vol. 17, no. 1, pp. 3133, 2011.

[96] Y. Gorin, K. Block, J. Hernandez et al., "NOX4 NAD(P)H oxidase mediates hypertrophy and fibronectin expression in the diabetic kidney," Journal of Biological Chemistry, vol. 280, no. 47, pp. 39616-39626, 2005.

[97] D. P. Jones, "Radical-free biology of oxidative stress," American Journal of Physiology, vol. 295, no. 4, pp. C849-C868, 2008.

[98] R. M. Liu, J. Choi, J. H. Wu et al., "Oxidative modification of nuclear mitogen-activated protein kinase phosphatase 1 is involved in transforming growth factor $\beta 1$-induced expression of plasminogen activator inhibitor 1 in fibroblasts," Journal of Biological Chemistry, vol. 285, no. 21, pp. 1623916247, 2010.

[99] K. Block, A. Eid, K. K. Griendling, D. Y. Lee, Y. Wittrant, and Y. Gorin, "NOX4 NAD $(\mathrm{P}) \mathrm{H}$ oxidase mediates Srcdependent tyrosine phosphorylation of PDK-1 in response to angiotensin II: role in mesangial cell hypertrophy and fibronectin expression," Journal of Biological Chemistry, vol. 283, no. 35, pp. 24061-24076, 2008.

[100] F. P. Bellinger, A. V. Raman, M. A. Reeves, and M. J. Berry, "Regulation and function of selenoproteins in human disease," Biochemical Journal, vol. 422, no. 1, pp. 11-22, 2009.

[101] S. C. Low, E. Grundner-Culemann, J. W. Harney, and M. J. Berry, "SECIS-SBP2 interactions dictate selenocysteine incorporation efficiency and selenoprotein hierarchy," EMBO Journal, vol. 19, no. 24, pp. 6882-6890, 2000.

[102] M. S. Crane, A. F. Howie, J. R. Arthur, F. Nicol, L. K. Crosley, and G. J. Beckett, "Modulation of thioredoxin reductase2 expression in EAhy926 cells: implications for endothelial selenoprotein hierarchy," Biochimica et Biophysica Acta, vol. 1790, no. 10, pp. 1191-1197, 2009.

[103] V. Mostert, I. Dreher, J. Köhrle, S. Wolff, and J. Abel, "Modulation of selenoprotein P expression by TGF- $\beta 1$ is mediated by Smad proteins," BioFactors, vol. 14, no. 1-4, pp. 135-142, 2001.

[104] V. Mostert, S. Wolff, I. Dreher, J. Köhrle, and J. Abel, "Identification of an element within the promoter of human selenoprotein P responsive to transforming growth factor- $\beta$," European Journal of Biochemistry, vol. 268, no. 23, pp. 61766181, 2001.

[105] E. Mezey, X. Liu, and J. J. Potter, "The combination of selenium and vitamin e inhibits type i collagen formation in cultured hepatic stellate cells," Biological Trace Element Research, vol. 140, no. 1, pp. 82-94, 2011.

[106] B. Contempre, O. Le Moine, J. E. Dumont, J. F. Denef, and M. C. Many, "Selenium deficiency and thyroid fibrosis. A key role for macrophages and transforming growth factor $\beta$ (TGF- $\beta$ )," Molecular and Cellular Endocrinology, vol. 124, no. 1-2, pp. 7-15, 1996.

[107] M. Ding, J. J. Potter, X. Liu, M. S. Torbenson, and E. Mezey, "Selenium supplementation decreases hepatic fibrosis in mice after chronic carbon tetrachloride administration," Biological Trace Element Research, vol. 133, no. 1, pp. 83-97, 2010.

[108] B. C. Pence, E. Delver, and D. M. Dunn, "Effects of dietary selenium on UVB-induced skin carcinogenesis and epidermal antioxidant status," Journal of Investigative Dermatology, vol. 102, no. 5, pp. 759-761, 1994.

[109] V. Diwadkar-Navsariwala, G. S. Prins, S. M. Swanson et al., "Selenoprotein deficiency accelerates prostate carcinogenesis in a transgenic model," Proceedings of the National Academy of Sciences of the United States of America, vol. 103, no. 21, pp. 8179-8184, 2006.

[110] M. Selenius, A. K. Rundlöf, E. Olm, A. P. Fernandes, and M. Björnstedt, "Selenium and the selenoprotein thioredoxin reductase in the prevention, treatment and diagnostics of cancer," Antioxidants and Redox Signaling, vol. 12, no. 7, pp. 867-880, 2010.

[111] A. J. Duffield-Lillico, B. L. Dalkin, M. E. Reid et al., "Selenium supplementation, baseline plasma selenium status and incidence of prostate cancer: an analysis of the complete treatment period of the Nutritional Prevention of Cancer Trial," BJU International, vol. 91, no. 7, pp. 608-612, 2003. 
[112] R. Hurst, L. Hooper, T. Norat et al., "Selenium and prostate cancer: systematic review and meta-analysis," The American Journal of Clinical Nutrition, vol. 96, no. 1, pp. 111-122, 2012.

[113] F. Murad, "Nitric oxide and cyclic GMP in cell signaling and drug development," The New England Journal of Medicine, vol. 355, no. 19, pp. 2003-2011, 2006.

[114] R. C. Rosen and J. B. Kostis, "Overview of phosphodiesterase 5 inhibition in erectile dysfunction," American Journal of Cardiology, vol. 92, no. 9, pp. 9M-18M, 2003.

[115] J. Houtchens, D. Martin, and J. R. Klinger, "Diagnosis and management of pulmonary arterial hypertension," Pulmonary Medicine, vol. 2011, Article ID 845864, 2011.

[116] M. Oelke, F. Giuliano, V. Mirone et al., "Monotherapy with tadalafil or tamsulosin similarly improved lower urinary tract symptoms suggestive of benign prostatic hyperplasia in an international, randomised, parallel, placebo-controlled clinical trial," European Urology, vol. 61, no. 5, pp. 917-925, 2012.

[117] J. I. Martínez-Salamanca, J. Carballido, I. Eardley et al., "Phosphodiesterase type 5 inhibitors in the management of non-neurogenic male lower urinary tract symptoms: Critical analysis of current evidence," European Urology, vol. 60, no. 3, pp. 527-535, 2011.

[118] M. Gacci, I. Eardley, F. Giuliano et al., "Critical analysis of the relationship between sexual dysfunctions and lower urinary tract symptoms due to benign prostatic hyperplasia," European Urology, vol. 60, no. 4, pp. 809-825, 2011.

[119] S. J. S. Grimsley, M. H. Khan, and G. E. Jones, "Mechanism of phosphodiesterase 5 inhibitor relief of prostatitis symptoms," Medical Hypotheses, vol. 69, no. 1, pp. 25-26, 2007.

[120] M. Takeda, "Effects of phosphodiesterase type 5 inhibitor on the contractility of prostate tissues and urethral pressure responses in a rat model of benign prostate hyperplasia: commentary," International Journal of Urology, vol. 14, no. 10, p. 951, 2007.

[121] S. Ückert, M. Sormes, G. Kedia et al., "Effects of phosphodiesterase inhibitors on tension induced by norepinephrine and accumulation of cyclic nucleotides in isolated human prostatic tissue," Urology, vol. 71, no. 3, pp. 526-530, 2008.

[122] A. J. Chu and J. K. Prasad, "Up-regulation by human recombinant transforming growth factor $\beta$-1 of collagen production in cultured dermal fibroblasts is mediated by the inhibition of nitric oxide signaling," Journal of the American College of Surgeons, vol. 188, no. 3, pp. 271-280, 1999.

[123] E. G. A. Valente, D. Vernet, M. G. Ferrini, A. Qian, J. Rajfer, and N. F. Gonzalez-Cadavid, "L-Arginine and phosphodiesterase (PDE) inhibitors counteract fibrosis in the Peyronie's fibrotic plaque and related fibroblast cultures," Nitric Oxide-Biology and Chemistry, vol. 9, no. 4, pp. 229244, 2003.

[124] C. Beyer, N. Reich, S. C. Schindler et al., "Stimulation of soluble guanylate cyclase reduces experimental dermal fibrosis," Annals of the Rheumatic Diseases, vol. 71, no. 6, pp. 10191026, 2012.

[125] D. Vernet, M. G. Ferrini, E. G. Valente et al., "Effect of nitric oxide on the differentiation of fibroblasts into myofibroblasts in the Peyronie's fibrotic plaque and in its rat model," Nitric Oxide-Biology and Chemistry, vol. 7, no. 4, pp. 262-276, 2002.

[126] M. G. Ferrini, S. Rivera, J. Moon, D. Vernet, J. Rajfer, and N. F. Gonzalez-Cadavid, "The genetic inactivation of inducible nitric oxide synthase (iNOS) intensifies fibrosis and oxidative stress in the penile corpora cavernosa in type 1 diabetes," Journal of Sexual Medicine, vol. 7, no. 9, pp. 3033-3044, 2010.
[127] D. Sun, Y. Wang, C. Liu et al., "Effects of nitric oxide on renal interstitial fibrosis in rats with unilateral ureteral obstruction," Life Sciences, vol. 90, no. 23-24, pp. 900-909, 2012.

[128] Y. Wang, S. Krämer, T. Loof et al., "Enhancing cGMP in experimental progressive renal fibrosis: Soluble guanylate cyclase stimulation vs. phosphodiesterase inhibition," American Journal of Physiology, vol. 290, no. 1, pp. F167-F176, 2006.

[129] Y. Sharkovska, P. Kalk, B. Lawrenz et al., "Nitric oxideindependent stimulation of soluble guanylate cyclase reduces organ damage in experimental low-renin and high-renin models," Journal of Hypertension, vol. 28, no. 8, pp. 16661675, 2010.

[130] S. Geschka, A. Kretschmer, Y. Sharkovska et al., "Soluble guanylate cyclase stimulation prevents fibrotic tissue remodeling and improves survival in salt-sensitive dahl rats," PLoS ONE, vol. 6, no. 7, Article ID e21853, 2011.

[131] A. Knorr, C. Hirth-Dietrich, C. Alonso-Alija et al., "Nitric oxide-independent activation of soluble guanylate cyclase by BAY 60-2770 in experimental liver fibrosis," ArzneimittelForschung/Drug Research, vol. 58, no. 2, pp. 71-80, 2008.

[132] B. Hohenstein, C. Daniel, S. Wittmann, and C. Hugo, "PDE5 inhibition impedes TSP- 1 expression, TGF- $\beta$ activation and matrix accumulation in experimental glomerulonephritis," Nephrology Dialysis Transplantation, vol. 23, no. 11, pp. 3427-3436, 2008.

[133] G. M. Rubanyi and P. M. Vanhoutte, "Superoxide anions and hyperoxia inactivate endothelium-derived relaxing factor," The American Journal of Physiology, vol. 250, no. 5, part 2, pp. H822-H827, 1986.

[134] N. D. Roe and J. Ren, "Nitric oxide synthase uncoupling: a therapeutic target in cardiovascular diseases," Vascular Pharmacology, vol. 57, no. 5-6, pp. 168-172, 2012.

[135] Y. M. Kim, H. Bergonia, and J. R. Lancaster Jr., "Nitrogen oxide-induced autoprotection in isolated rat hepatocytes," FEBS Letters, vol. 374, no. 2, pp. 228-232, 1995.

[136] E. A. Jaimes, C. Sweeney, and L. Raij, "Effects of the reactive oxygen species hydrogen peroxide and hypochlorite on endothelial nitric oxide production," Hypertension, vol. 38, no. 4, pp. 877-883, 2001.

[137] E. P. Wei and H. A. Kontos, " $\mathrm{H}_{2} \mathrm{O}_{2}$ and endotheliumdependent cerebral arteriolar dilation. Implications for the identity of endothelium-derived relaxing factor generated by acetylcholine," Hypertension, vol. 16, no. 2, pp. 162-169, 1990.

[138] C. Zenzmaier, J. Kern, N. Sampson et al., "Phosphodiesterase type 5 inhibition reverts prostate fibroblast-to-myofibroblast trans-differentiation," Endocrinology, 2012. In press.

[139] J. Ying, X. Tong, D. R. Pimentel et al., "Cysteine-674 of the sarco/endoplasmic reticulum calcium ATPase is required for the inhibition of cell migration by nitric oxide," Arteriosclerosis, Thrombosis, and Vascular Biology, vol. 27, no. 4, pp. 783790, 2007.

[140] T. Adachi, R. Matsui, R. M. Weisbrod, S. Najibi, and R. A. Cohen, "Reduced sarco/endoplasmic reticulum $\mathrm{Ca}^{2+}$ uptake activity can account for the reduced response to NO, but not sodium nitroprusside, in hypercholesterolemic rabbit aorta," Circulation, vol. 104, no. 9, pp. 1040-1045, 2001.

[141] X. Tong, X. Hou, D. Jourd'Heuil, R. M. Weisbrod, and R. A. Cohen, "Upregulation of NOX4 by TGF $\beta 1$ oxidizes SERCA and inhibits $\mathrm{NO}$ in arterial smooth muscle of the prediabetic zucker rat," Circulation Research, vol. 107, no. 8, pp. 975-983, 2010 . 
[142] X. Tong and K. Schröder, "NADPH oxidases are responsible for the failure of nitric oxide to inhibit migration of smooth muscle cells exposed to high glucose," Free Radical Biology and Medicine, vol. 47, no. 11, pp. 1578-1583, 2009.

[143] S. Wedgwood, R. H. Steinhorn, M. Bunderson et al., "Increased hydrogen peroxide downregulates soluble guanylate cyclase in the lungs of lambs with persistent pulmonary hypertension of the newborn," American Journal of Physiology, vol. 289, no. 4, pp. L660-L666, 2005.

[144] C. Gerassimou, A. Kotanidou, Z. Zhou, D. D. C. Simoes, C. Roussos, and A. Papapetropoulos, "Regulation of the expression of soluble guanylyl cyclase by reactive oxygen species," British Journal of Pharmacology, vol. 150, no. 8, pp. 10841091, 2007.

[145] S. Meurer, S. Pioch, S. Gross, and W. Müller-Esterl, "Reactive oxygen species induce tyrosine phosphorylation of and Src kinase recruitment to NO-sensitive guanylyl cyclase," Journal of Biological Chemistry, vol. 280, no. 39, pp. 33149-33156, 2005.

[146] K. S. Murthy, "Inhibitory phosphorylation of soluble guanylyl cyclase by muscarinic $\mathrm{m} 2$ receptors via $\mathrm{G} \beta \gamma$-dependent activation of c-Src kinase," Journal of Pharmacology and Experimental Therapeutics, vol. 325, no. 1, pp. 183-189, 2008.

[147] N. Abdelaziz, F. Colombo, I. Mercier, and A. Calderone, "Nitric oxide attenuates the expression of transforming growth factor- $\beta 3$ mRNA in rat cardiac fibroblasts via destabilization," Hypertension, vol. 38, no. 2, pp. 261-266, 2001.

[148] A. Bellocq, E. Azoulay, S. Marullo et al., "Reactive oxygen and nitrogen intermediates increase transforming growth factor$\beta 1$ release from human epithelial alveolar cells through two different mechanisms," American Journal of Respiratory Cell and Molecular Biology, vol. 21, no. 1, pp. 128-136, 1999.

[149] P. R. Bachiller, H. Nakanishi, and J. D. Roberts, "Transforming growth factor- $\beta$ modulates the expression of nitric oxide signaling enzymes in the injured developing lung and in vascular smooth muscle cells," American Journal of Physiology, vol. 298, no. 3, pp. L324-L334, 2010.

[150] A. Douglass, K. Wallace, R. Parr et al., "Antibody-targeted myofibroblast apoptosis reduces fibrosis during sustained liver injury," Journal of Hepatology, vol. 49, no. 1, pp. 88-98, 2008.

[151] M. Bocchino, S. Agnese, E. Fagone et al., "Reactive oxygen species are required for maintenance and differentiation of primary lung fibroblasts in idiopathic pulmonary fibrosis," PLoS ONE, vol. 5, no. 11, Article ID e14003, 2010.

[152] M. C. Vozenin-Brotons, V. Sivan, N. Gault et al., "Antifibrotic action of $\mathrm{Cu} / \mathrm{Zn}$ SOD is mediated by TGF- $\beta 1$ repression and phenotypic reversion of myofibroblasts," Free Radical Biology and Medicine, vol. 30, no. 1, pp. 30-42, 2001.

[153] O. Maltseva, P. Folger, D. Zekaria, S. Petridou, and S. K. Masur, "Fibroblast growth factor reversal of the corneal myofibroblast phenotype," Investigative Ophthalmology and Visual Science, vol. 42, no. 11, pp. 2490-2495, 2001.

[154] M. G. Ferrini, I. Kovanecz, G. Nolazco, J. Rajfer, and N. F. Gonzalez-Cadavid, "Effects of long-term vardenafil treatment on the development of fibrotic plaques in a rat model of Peyronie's disease," BJU International, vol. 97, no. 3, pp. 625-633, 2006.

[155] A. Leask, "Potential therapeutic targets for cardiac fibrosis: TGF $\beta$, angiotensin, endothelin, CCN2, and PDGF, partners in fibroblast activation," Circulation Research, vol. 106, no. 11, pp. 1675-1680, 2010.
[156] C. L. Miller, Y. Cai, M. Oikawa et al., "Cyclic nucleotide phosphodiesterase 1A: a key regulator of cardiac fibroblast activation and extracellular matrix remodeling in the heart," Basic Research in Cardiology, vol. 106, no. 6, pp. 1023-1039, 2011. 


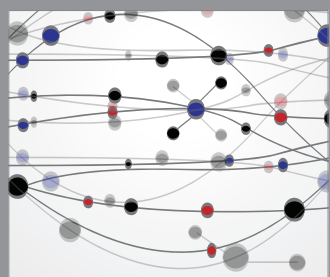

The Scientific World Journal
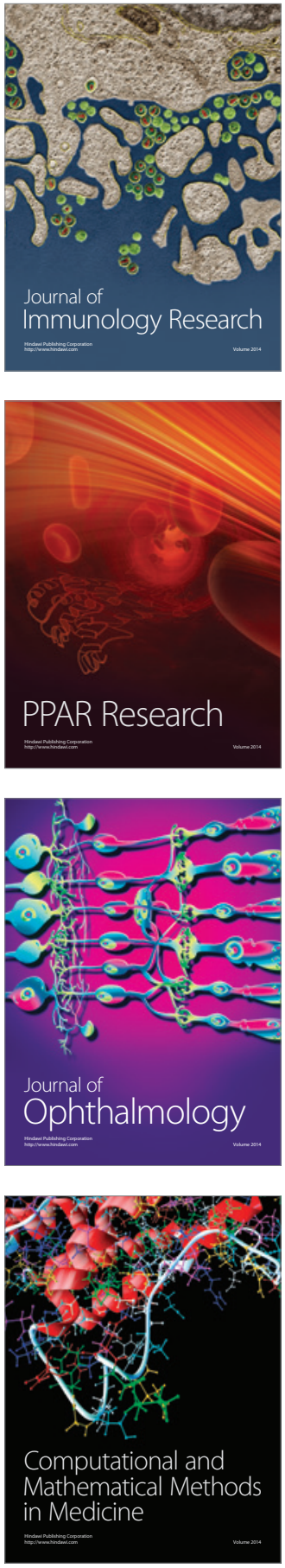

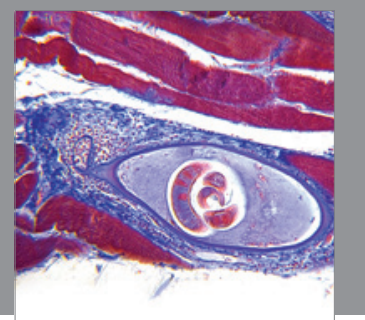

Gastroenterology

Research and Practice
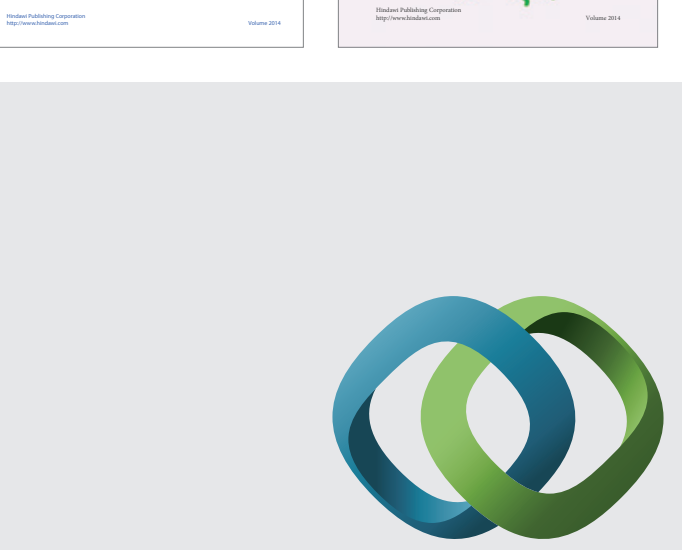

\section{Hindawi}

Submit your manuscripts at

http://www.hindawi.com
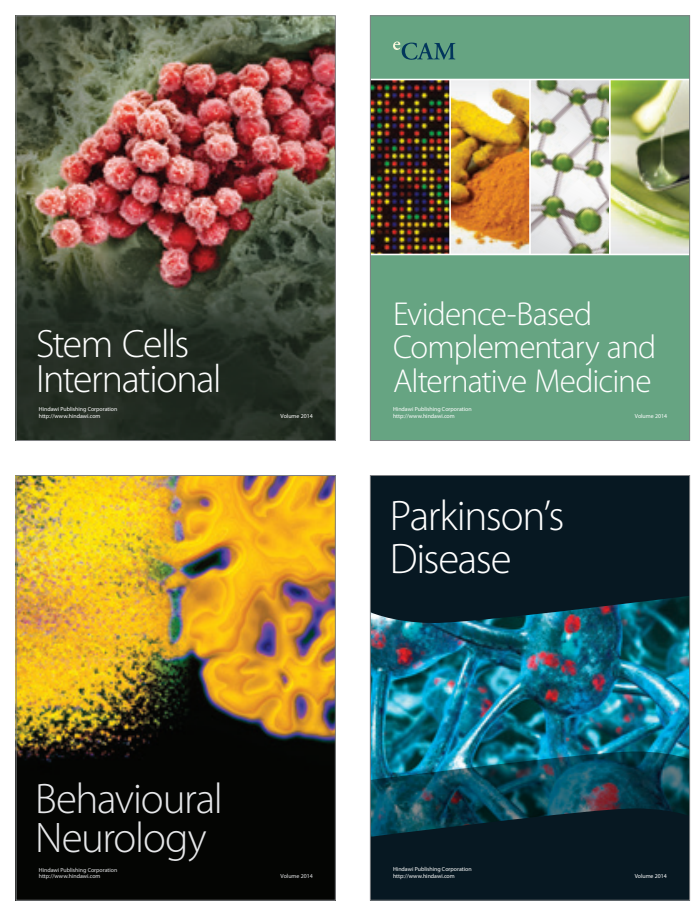

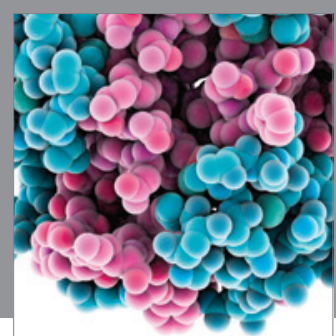

Journal of
Diabetes Research

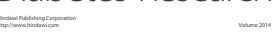

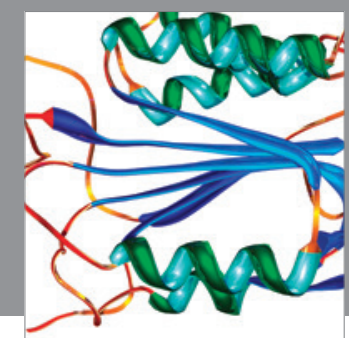

Disease Markers
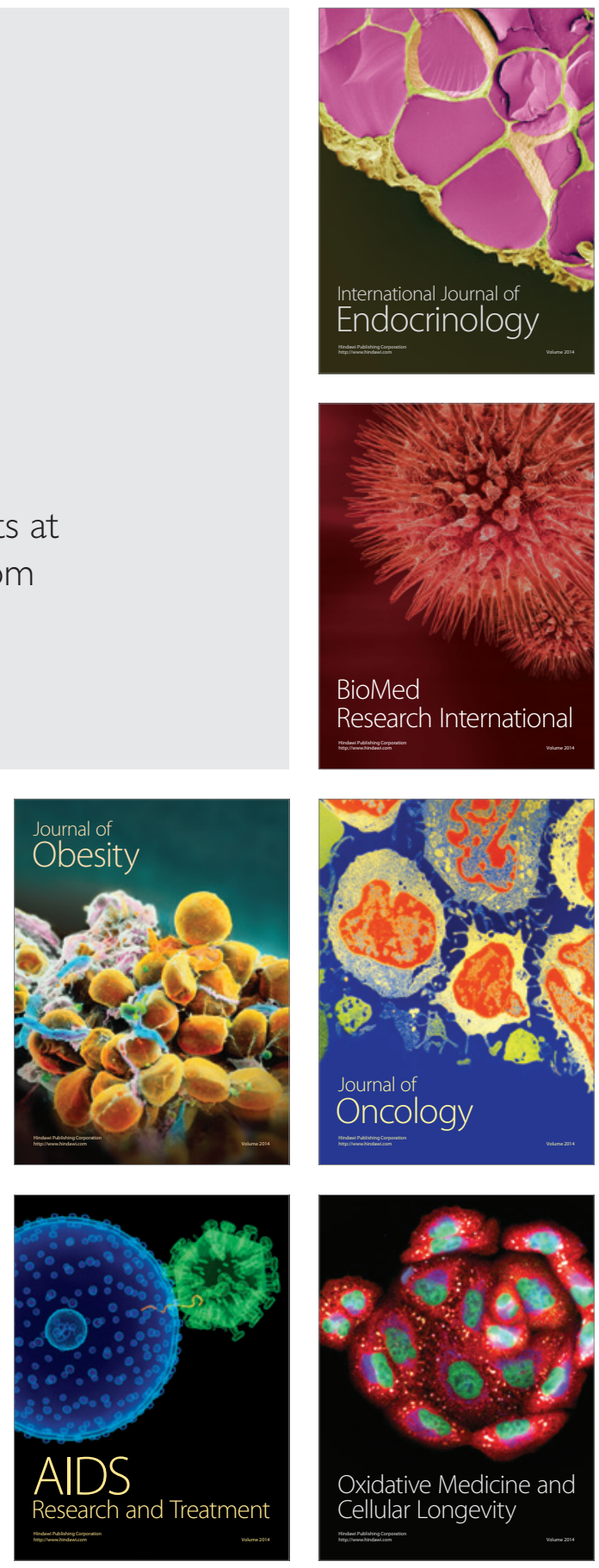